TRANSACTIONS OF THE

AMERICAN MATHEMATICAL SOCIETY

Volume 355, Number 8, Pages 3253-3275

S 0002-9947(03)03284-7

Article electronically published on April 8, 2003

\title{
CONSTRUCTIONS PRESERVING HILBERT SPACE UNIFORM EMBEDDABILITY OF DISCRETE GROUPS
}

\author{
MARIUS DADARLAT AND ERIK GUENTNER
}

\begin{abstract}
Uniform embeddability (in a Hilbert space), introduced by Gromov, is a geometric property of metric spaces. As applied to countable discrete groups, it has important consequences for the Novikov conjecture. Exactness, introduced and studied extensively by Kirchberg and Wassermann, is a functional analytic property of locally compact groups. Recently it has become apparent that, as properties of countable discrete groups, uniform embeddability and exactness are closely related. We further develop the parallel between these classes by proving that the class of uniformly embeddable groups shares a number of permanence properties with the class of exact groups. In particular, we prove that it is closed under direct and free products (with and without amalgam), inductive limits and certain extensions.
\end{abstract}

\section{INTRODUCTION}

Gromov introduced the notion of uniform embeddability of metric spaces, and suggested that finitely generated discrete groups that are uniformly embeddable in a Hilbert space, when viewed as metric spaces, might satisfy the Novikov conjecture [12], [10]. Yu proved that this is indeed the case [20], [18].

Kirchberg and Wassermann defined the notion of exactness of a locally compact group in terms of the behavior of its reduced crossed product functor. Subsequently, they developed the main properties of exact groups. In particular, they showed that in the case of a countable discrete group, exactness can be reformulated entirely in terms of the reduced $C^{*}$-algebra [14], that is, that exactness is a property of the harmonic analysis of the left regular representation of such a group.

The starting point of this work is the startling fact that for countable discrete groups, uniform embeddability (in a Hilbert space, a geometric property) and exactness (an analytic property) are closely related. The first indications of the relationship between uniform embeddability and exactness are found in the work of Guentner and Kaminker [13; these preliminary steps were quickly expanded by Ozawa [16], Anantharaman-Delaroche [2], and others.

We are concerned with uniformly embeddable groups. Our main results are outlined in the following theorem (more precise statements follow in later sections),

Received by the editors July 22, 2002 and, in revised form, December 26, 2002.

2000 Mathematics Subject Classification. Primary 46L89, 20F65.

The first author was supported in part by an MSRI Research Professorship and NSF Grant DMS-9970223. The second author was supported in part by an MSRI Postdoctoral Fellowship and NSF Grant DMS-0071402. 
which summarizes the basic permanence properties of the class of uniformly embeddable groups. Observe that the properties described are all shared by the class of countable discrete exact groups [15]. Indeed, in each case it is possible to give a unified account of the results for uniform embeddability and exactness; in some cases our methods provide alternate proofs of the known results concerning exactness.

Theorem. The class of countable discrete groups that are uniformly embeddable in a Hilbert space is closed under subgroups and products, direct limits, free products with amalgam, and extensions by exact groups.

The fact that subgroups and products of uniformly embeddable groups are again uniformly embeddable is elementary and quite well known; they are included in the statement for completeness.

The other properties are more difficult to establish. It is possible to construct a uniform embedding of a free product (without amalgam) directly from uniform embeddings of the factors [5]. On the other hand, the corresponding result for free products with amalgam is considerably more difficult, in view of the fact that the common subgroup of the amalgam can introduce considerable distortion into the product. Our proof is based on a suitable adaptation of an argument given by $\mathrm{Tu}$ in his work on Property A [19]; although we are not able to verify a number of assertions concerning the metric defined in Section 9 in Tu's paper, we are able to adapt his arguments to the present context. The proof we give works equally well for countable exact groups (see Proposition 6.8 and Theorem 6.9), and is unrelated to Dykema's original proof that the class of countable exact groups is closed under free products with amalgam [9], [8]. Again, in the case without amalgam a considerably simpler proof of this fact is now available [5].

The general problem of uniform embeddability of extensions is intriguing. Our proof that the class of uniformly embeddable groups is closed under extensions by exact groups is inspired by the argument of Anantharaman-Delaroche and Renault showing that the class of countable exact groups is closed under extensions [3]. It is unknown whether the class of uniformly embeddable groups is closed under general extensions; even the case of a central extension of $\mathbb{Z}$ by a uniformly embeddable group remains open. At present, the behavior with respect to extensions provides the best possibility of distinguishing the classes of uniformly embeddable and exact groups.

We draw two immediate corollaries. Since they are peripheral to our study we will not establish notation or provide the relevant definitions; rather, we provide references.

Corollary. The class of countable discrete groups that are uniformly embeddable in a Hilbert space is closed under the formation of HNN extensions.

Proof. An HNN extension is built from free products with amalgam, direct limits, and a semi-direct product by $\mathbb{Z}$, which is exact [17, [4. (See [6] for related results.)

Corollary. The fundamental group of a graph of countable discrete groups is uniformly embeddable in a Hilbert space if and only if each of the groups is uniformly embeddable in a Hilbert space. 
Proof. Each constituent group is a subgroup of the fundamental group. Conversely, the fundamental group of a graph of groups is built from free products with amalgam, HNN extensions and direct limits [17], [4]. (See [1] for related remarks.)

\section{BACKGROUND}

Let $X$ and $Y$ be metric spaces, with metrics $d_{X}$ and $d_{Y}$, respectively. A function $F: X \rightarrow Y$ is a uniform embedding if there exist non-decreasing functions $\rho_{ \pm}$: $\mathbb{R}_{+} \rightarrow \mathbb{R}_{+}$such that $\lim _{t \rightarrow \infty} \rho_{ \pm}(t)=\infty$ and such that

$$
\rho_{-}\left(d_{X}\left(x, x^{\prime}\right)\right) \leq d_{Y}\left(F(x), F\left(x^{\prime}\right)\right) \leq \rho_{+}\left(d_{X}\left(x, x^{\prime}\right)\right), \quad \text { for all } x, x^{\prime} \in X .
$$

The space $X$ is uniformly embeddable if there exists a uniform embedding $F$ of $X$ into a Hilbert space $\mathcal{H}$. Uniform embeddability in a real Hilbert space is equivalent to uniform embeddability in a complex Hilbert space; henceforth we shall deal only with real Hilbert spaces. Obviously, if $X$ is countable we may assume that the Hilbert space is separable.

A metric space $X$ is locally finite if for every $x \in X$ and $R>0$ the metric ball with center $x$ and radius $R$ is finite. In this case the metric is called proper. A locally finite metric space is discrete (as a topological space in the metric topology). In the case of locally finite metric spaces there are a number of equivalent formulations of uniform embeddability [7], 13]; to these we add the following simple extension, which applies to any metric space and which will be our fundamental criterion for uniform embeddability.

Proposition 2.1. Let $X$ be a metric space. Then $X$ is uniformly embeddable if and only if for every $R>0$ and $\varepsilon>0$ there exists a Hilbert space valued map $\xi: X \rightarrow \mathcal{H},\left(\xi_{x}\right)_{x \in X}$, such that $\left\|\xi_{x}\right\|=1$ for all $x \in X$, and such that

(i) $\sup \left\{\left\|\xi_{x}-\xi_{x^{\prime}}\right\|: d\left(x, x^{\prime}\right) \leq R, x, x^{\prime} \in X\right\} \leq \varepsilon$,

(ii) $\lim _{S \rightarrow \infty} \sup \left\{\left|\left\langle\xi_{x}, \xi_{x^{\prime}}\right\rangle\right|: d\left(x, x^{\prime}\right) \geq S, x, x^{\prime} \in X\right\}=0$.

These conditions may be replaced by

(iii) $\sup \left\{\left|1-\left\langle\xi_{x}, \xi_{x^{\prime}}\right\rangle\right|: d\left(x, x^{\prime}\right) \leq R, x, x^{\prime} \in X\right\} \leq \varepsilon$,

(iv) $\lim _{S \rightarrow \infty} \inf \left\{\left\|\xi_{x}-\xi_{x^{\prime}}\right\|: d\left(x, x^{\prime}\right) \geq S, x, x^{\prime} \in X\right\}=2$,

respectively.

Remark. We refer to (i) and (iii) collectively as the convergence condition; similarly, we refer to (ii) and (iv) collectively as the support condition.

Proof. The interchangeability of (i) $\Leftrightarrow$ (iii) and (ii) $\Leftrightarrow$ (iv) follows from the simple observation that for unit vectors $\xi, \eta \in \mathcal{H}$ we have $\|\xi-\eta\|^{2}=2-2\langle\xi, \eta\rangle$.

Assume that $X$ is uniformly embeddable and let $F: X \rightarrow \mathcal{H}$ be a uniform embedding in a real Hilbert space $\mathcal{H}$. Let

$$
\operatorname{Exp}(\mathcal{H})=\mathbb{R} \oplus \mathcal{H} \oplus(\mathcal{H} \otimes \mathcal{H}) \oplus(\mathcal{H} \otimes \mathcal{H} \otimes \mathcal{H}) \oplus \cdots
$$

and define $\operatorname{Exp}: \mathcal{H} \rightarrow \operatorname{Exp}(\mathcal{H})$ by

$$
\operatorname{Exp}(\zeta)=1 \oplus \zeta \oplus\left(\frac{1}{\sqrt{2 !}} \zeta \otimes \zeta\right) \oplus\left(\frac{1}{\sqrt{3 !}} \zeta \otimes \zeta \otimes \zeta\right) \oplus \cdots
$$

Note that $\left\langle\operatorname{Exp}(\zeta), \operatorname{Exp}\left(\zeta^{\prime}\right)\right\rangle=e^{\left\langle\zeta, \zeta^{\prime}\right\rangle}$, for all $\zeta, \zeta^{\prime} \in \mathcal{H}$. For $t>0$ define

$$
\xi_{x}=e^{-t\|F(x)\|^{2}} \operatorname{Exp}(\sqrt{2 t} F(x)) .
$$


It is easily verified that $\left\langle\xi_{x}, \xi_{x^{\prime}}\right\rangle=e^{-t\left\|F(x)-F\left(x^{\prime}\right)\right\|^{2}}$. Consequently, for all $x, x^{\prime} \in X$ we have $\left\|\xi_{x}\right\|=1$, and

$$
e^{-t \rho_{+}\left(d\left(x, x^{\prime}\right)\right)^{2}} \leq\left\langle\xi_{x}, \xi_{x^{\prime}}\right\rangle \leq e^{-t \rho_{-}\left(d\left(x, x^{\prime}\right)\right)^{2}} .
$$

Letting $t=\varepsilon\left(1+\rho_{+}(R)^{2}\right)^{-1}$, it is easy to verify the conditions (iii) and (ii) above.

Conversely, assume that $X$ satisfies the conditions in the second part of the statement. There exist a sequence of maps $\eta_{n}: X \rightarrow \mathcal{H}_{n}$ and a sequence of numbers $S_{0}=0<S_{1}<S_{2}<\ldots$, increasing to infinity, such that for every $n \geq 1$ and every $x, x^{\prime} \in X$,

(i) $\left\|\eta_{n}(x)\right\|=1$,

(ii) $\left\|\eta_{n}(x)-\eta_{n}\left(x^{\prime}\right)\right\| \leq 1 / n$, provided $d\left(x, x^{\prime}\right) \leq \sqrt{n}$,

(iii) $\left\|\eta_{n}(x)-\eta_{n}\left(x^{\prime}\right)\right\| \geq 1$, provided $d\left(x, x^{\prime}\right) \geq S_{n}$.

Choose a base point $x_{0} \in X$ and define $F: X \rightarrow \bigoplus_{n=1}^{\infty} \mathcal{H}_{n}$ by

$$
F(x)=\frac{1}{2}\left(\eta_{1}(x)-\eta_{1}\left(x_{0}\right) \oplus \eta_{2}(x)-\eta_{2}\left(x_{0}\right) \oplus \cdots\right) .
$$

It is not hard to verify that $F$ is well defined and

$$
\rho_{-}\left(d\left(x, x^{\prime}\right)\right) \leq\left\|F(x)-F\left(x^{\prime}\right)\right\| \leq d\left(x, x^{\prime}\right)+1, \quad \text { for all } x, x^{\prime} \in X,
$$

where $\rho_{-}=\frac{1}{2} \sum_{n=1}^{\infty} \sqrt{n-1} \chi_{\left[S_{n-1}, S_{n}\right)}$, and the $\chi_{\left[S_{n-1}, S_{n}\right)}$ are the characteristic functions of the sets $\left[S_{n-1}, S_{n}\right)$.

Indeed, let $x, x^{\prime} \in X$. If $n$ is such that $\sqrt{n-1} \leq d\left(x, x^{\prime}\right)<\sqrt{n}$, we have

$$
\begin{aligned}
\left\|F(x)-F\left(x^{\prime}\right)\right\|^{2} & =\frac{1}{4} \sum_{i \leq n-1}\left\|\eta_{i}(x)-\eta_{i}\left(x^{\prime}\right)\right\|^{2}+\frac{1}{4} \sum_{i \geq n}\left\|\eta_{i}(x)-\eta_{i}\left(x^{\prime}\right)\right\|^{2} \\
& \leq(n-1)+\frac{1}{4} \sum_{i \geq n} \frac{1}{i^{2}} \leq d\left(x, x^{\prime}\right)^{2}+1 .
\end{aligned}
$$

Similarly, if $n$ is such that $S_{n-1} \leq d\left(x, x^{\prime}\right)<S_{n}$, we have

$$
\left\|F(x)-F\left(x^{\prime}\right)\right\|^{2} \geq \frac{1}{4} \sum_{i \leq n-1}\left\|\eta_{i}(x)-\eta_{i}\left(x^{\prime}\right)\right\|^{2} \geq \frac{n-1}{4}=\rho_{-}\left(d\left(x, x^{\prime}\right)\right)^{2} .
$$

Remark. Straightforward modifications of the above argument produce a uniform embedding $F$ satisfying the sharper estimate $\left\|F(x)-F\left(x^{\prime}\right)\right\| \leq d\left(x, x^{\prime}\right)+\delta$, for an arbitrarily chosen $\delta>0$; simply replace the $1 / n$ in (ii) by $\delta / 2^{n}$.

Two metrics $d$ and $d^{\prime}$ on the set $X$ are coarsely equivalent if for every $R>0$ there exists an $S>0$ such that the $d-$ metric ball with center $x$ and radius $R$ is contained in the $d^{\prime}$-metric ball with center $x$ and radius $S$; and conversely. Equivalently, two metrics on $X$ are coarsely equivalent if the identity map $X \rightarrow X$ is a uniform embedding.

Proposition 2.2. Let $d$ and $d^{\prime}$ be coarsely equivalent metrics on $X$. Then $X$ is uniformly embeddable with respect to $d$ if and only if it is uniformly embeddable with respect to $d^{\prime}$.

Proof. If two maps are uniform embeddings, so is their composition.

Remark. Below we require only the fact that if $Y \rightarrow \mathcal{H}$ and $X \rightarrow Y$ are uniform embeddings, then the composite $X \rightarrow \mathcal{H}$ is a uniform embedding. In other words, we do not need to know that $X$ and $Y$ are coarsely equivalent to conclude the uniform embeddability of $X$ from that of $Y$. 
Let $\Gamma$ be a countable discrete group. A length function on $\Gamma$ is a nonnegative, real-valued function $l$ satisfying, for all $a$ and $b$ in $\Gamma$,

(i) $l(a b) \leq l(a)+l(b)$,

(ii) $l\left(a^{-1}\right)=l(a)$,

(iii) $l(a)=0$ if and only if $a=1$.

A length function $l$ is proper if for all $C>0$ the subset $l^{-1}([0, C]) \subset \Gamma$ is finite. One can construct an integer-valued proper length function on $\Gamma$ as follows. Let $S$ be a symmetric set of generators of $\Gamma$. Let $l_{0}: S \rightarrow \mathbb{N}$ be a proper function satisfying (ii) and (iii) above. Then

$$
l(a)=\inf \left\{l_{0}\left(a_{1}\right)+\cdots+l_{0}\left(a_{n}\right): a=a_{1} \cdots a_{n}, a_{i} \in S\right\}
$$

is a proper length function on $\Gamma$. Given a length function $l$, we define a metric $d$ by $d(a, b)=l\left(a^{-1} b\right)$. A metric constructed in this way from a length function is left-invariant in the sense that $d(c a, c b)=d(a, b)$, for all $a, b$ and $c \in \Gamma$. Conversely, every left-invariant metric arises in this way from a length function. A length function is proper if and only if the corresponding (left-invariant) metric has bounded geometry. Recall that a metric space $X$ has bounded geometry if for every $R>0$, there is a uniform bound on the number of elements in the balls of radius $R$ in $X$.

We require the following well-known proposition (compare [19, Lemma 2.1]).

Proposition 2.3. Let $\Gamma$ be a countable discrete group and let $d$ and $d^{\prime}$ be metrics on $\Gamma$ associated to proper length functions $l$ and $l^{\prime}$, respectively. Then $d$ and $d^{\prime}$ are coarsely equivalent.

Proof. Since the metrics are left-invariant, it suffices to consider the containment, as in the definition, of balls centered at the identity element. By symmetry it suffices to show that for every $R>0$ there exists an $S>0$ such that, for all $a \in \Gamma$, if $l(a)<R$, then $l^{\prime}(a)<S$. But, since $l$ is proper, this is obvious.

As a consequence of the previous two propositions, uniform embeddability of a countable discrete group $\Gamma$ is independent of the particular proper length function used to define its metric. Consequently, we systematically omit reference to a specific length function or metric in the statements of all results and say simply $\Gamma$ is uniformly embeddable to mean that $\Gamma$ is uniformly embeddable in a Hilbert space for some (equivalently all) left-invariant proper metric(s).

Finally, we draw two simple consequences. An action of a discrete group on a locally finite metric space $X$ is proper if for every bounded subset $B \subset X$ the set $\{a \in \Gamma: a \cdot B \cap B \neq \emptyset\}$ is finite. Equivalently, for every $x \in X$ and $R \geq 0$ the set $\left\{a \in \Gamma: a \cdot x \in B_{R}(x)\right\}$ is finite. Observe that a free action of a discrete group on a locally finite metric space is proper.

Corollary 2.4. Let $\Gamma$ be a countable discrete group equipped with a left-invariant proper metric. Let $X$ be a locally finite metric space equipped with a free isometric action of $\Gamma$. Then the inclusion $\Gamma \rightarrow X$ as an orbit is a uniform embedding.

Proof. Let $x_{0} \in X$. Since the action of $\Gamma$ on $X$ is by isometries, $l(a)=d_{X}\left(a \cdot x_{0}, x_{0}\right)$ defines a length function on $\Gamma$. Since the action is free and $X$ is locally finite, $l$ is a proper length function. Let $d$ be the left-invariant metric associated to $l$.

According to the previous proposition, the original metric on $\Gamma$ is coarsely equivalent to $d$, which is precisely what was to be proved. 
Corollary 2.5. Let $X$ and $\Gamma$ be as in the statement of the previous corollary. If $X$ is uniformly embeddable, then so is $\Gamma$.

Property A is a condition on metric spaces introduced by $\mathrm{Yu} 20$. We will work with the following characterization of Property A.

Proposition 2.6 ([19]). A discrete metric space $X$ with bounded geometry has Property $A$ if and only if for every $R>0$ and $\varepsilon>0$ there exist an $S>0$ and a Hilbert space valued function $\xi: X \rightarrow \mathcal{H}$ such that for all $x, x^{\prime} \in X$ we have $\left\|\xi_{x}\right\|=1$, and

(i) $d\left(x, x^{\prime}\right) \leq R \Rightarrow\left\|\xi_{x}-\xi_{x^{\prime}}\right\| \leq \varepsilon$,

(ii) $d\left(x, x^{\prime}\right) \geq S \Rightarrow\left\langle\xi_{x}, \xi_{x^{\prime}}\right\rangle=0$.

Equivalently, for every $R>0$ and $\varepsilon>0$ there exist an $S>0$ and $\xi: X \rightarrow l^{2}(X)$ such that for all $x, x^{\prime} \in X$ we have $\left\|\xi_{x}\right\|=1$, (i) as above, and

(iii) $\operatorname{supp} \xi_{x} \subset B_{S}(x)$.

Remark. As in the case of uniform embeddability, we refer to (i) as the convergence condition and to (ii) and (iii) collectively as the support condition.

It is clear from the proposition that Property A is invariant under coarse equivalence. We refer the reader to [15] for an introduction to exact groups. Our interest in Proposition 2.6 is motivated by the following result, inspired by [13].

Theorem 2.7 (16], and also [2]). A countable discrete group $\Gamma$ is exact if and only if $\Gamma$ has Property $A$ with respect to some (every) left-invariant proper metric.

In analogy with Corollary 2.5 we have the following result, in which we do not assume that $X$ itself has Property A.

Corollary 2.8. Let $\Gamma$ be a countable discrete group. Assume that $\Gamma$ acts freely and isometrically on a locally finite metric space $X$ satisfying the convergence and support conditions of Proposition 2.6. Then $\Gamma$ is exact.

Proof. Include $\Gamma \subset X$ as an orbit. The convergence and support conditions of Proposition 2.6 pass from $X$ to the subspace $\Gamma$. Since $\Gamma$ has bounded geometry, Proposition 2.6 applies.

\section{Limits}

We begin to establish the closure properties of the class of countable discrete uniformly embeddable groups. In this section we treat direct limits, our main result being the following proposition.

Proposition 3.1. Let $\Gamma$ be the limit of a directed system of countable discrete groups $G_{1} \rightarrow G_{2} \rightarrow G_{3} \rightarrow \cdots$ in which the maps $G_{n} \rightarrow G_{n+1}$ are injective. If each of the groups $G_{n}$ is uniformly embeddable, then so is $\Gamma$.

Proof. The proof is based on a method of extending Hilbert space valued functions from a subgroup to an ambient group. Specifically, let $\xi: G \rightarrow \mathcal{H}$ be a Hilbert space valued function on a subgroup $G$ of a countable discrete group $\Gamma$. Choose and fix a family of coset representatives $x \in X \subset \Gamma$ for $\Gamma / G$; having done so, we can express each element $a \in \Gamma$ uniquely as a product $x_{a} g_{a}$, where $x_{a} \in X$ and $g_{a} \in G$. The extension $\widehat{\xi}$ of $\xi$ is defined by

$$
\widehat{\xi}: \Gamma \rightarrow \mathcal{H} \otimes l^{2}(\Gamma / G) \cong \mathcal{H} \otimes l^{2}(X), \quad \widehat{\xi}_{a}=\xi_{g_{a}} \otimes \delta_{a G} \cong \xi_{g_{a}} \otimes \delta_{x_{a}} .
$$


Now let $\Gamma$ be a direct limit as in the statement of the theorem. Equip $\Gamma$ with a proper length function $l_{\Gamma}$ and associated metric $d_{\Gamma}$. Metrize each of the subgroups $G_{n}$ as subspaces of $\Gamma$; the metric and length function on $G_{n}$ are simply the restriction of $d_{\Gamma}$ and $l_{\Gamma}$. Observe that for $a, b \in \Gamma$ we have

$$
a^{-1} b \in G_{n} \Leftrightarrow a G_{n}=b G_{n} \Leftrightarrow x_{a}=x_{b} \in \Gamma,
$$

and that, in this case,

$$
d_{G_{n}}\left(g_{a}, g_{b}\right)=d_{\Gamma}\left(g_{a}, g_{b}\right)=d_{\Gamma}\left(x_{a} g_{a}, x_{b} g_{b}\right)=d_{\Gamma}(a, b) .
$$

We show that $\Gamma$ satisfies the convergence and support conditions of Proposition 2.1 Let $\varepsilon>0$ and $R>0$ be given. Obtain $n$ such that if $a \in \Gamma$ has $l_{\Gamma}(a) \leq R$, then $a \in G_{n}$; this is possible because the length function $l_{\Gamma}$ on $\Gamma$ is assumed to be proper. According to the criterion for uniform embeddability, obtain a Hilbert space valued function $\xi: G_{n} \rightarrow \mathcal{H}$ such that for all $g, h \in G_{n}$ we have $\left\|\xi_{g}\right\|=1$ and

(i) if $d_{G_{n}}(g, h) \leq R$, then $\left\|\xi_{g}-\xi_{h}\right\|<\varepsilon$;

(ii) $\forall \hat{\varepsilon}>0 \exists S>0$ such that if $d_{G_{n}}(g, h) \geq S$, then $\left|\left\langle\xi_{g}, \xi_{h}\right\rangle\right|<\hat{\varepsilon}$.

Let $\widehat{\xi}$ be the extension of $\xi$ to $\Gamma$ defined above. Clearly, $\left\|\widehat{\xi}_{a}\right\|=1$ for all $a \in \Gamma$, and it remains to verify the conditions of Proposition 2.1 Let $a, b \in \Gamma$. For the convergence condition, assume that $l_{\Gamma}\left(a^{-1} b\right)=d_{\Gamma}(a, b) \leq R$. By our choice of $n$ we have $a^{-1} b \in G_{n}$ and, according to (3) $), d_{G_{n}}\left(g_{a}, g_{b}\right)=d_{\Gamma}(a, b) \leq R$. Therefore,

$$
\left\|\widehat{\xi}_{a}-\widehat{\xi}_{b}\right\|=\left\|\xi_{g_{a}} \otimes \delta_{a G_{n}}-\xi_{g_{b}} \otimes \delta_{b G_{n}}\right\|=\left\|\xi_{g_{a}}-\xi_{g_{b}}\right\|<\varepsilon .
$$

For the support condition let $\hat{\varepsilon}>0$, obtain $S$ as in (ii) above, and assume $d_{\Gamma}(a, b) \geq$ $S$. According to

$$
\left\langle\widehat{\xi}_{a}, \widehat{\xi}_{b}\right\rangle=\left\langle\xi_{g_{a}}, \xi_{g_{b}}\right\rangle\left\langle\delta_{a G_{n}}, \delta_{b G_{n}}\right\rangle= \begin{cases}\left\langle\xi_{g_{a}}, \xi_{g_{b}}\right\rangle, & \text { if } a G_{n}=b G_{n} \\ 0, & \text { otherwise }\end{cases}
$$

we may assume $a^{-1} b \in G_{n}$. But then, according to (3) again, $d_{G_{n}}\left(g_{a}, g_{b}\right)=d_{\Gamma}(a, b)$ and $\left|\left\langle\widehat{\xi}_{a}, \widehat{\xi}_{b}\right\rangle\right|<\hat{\varepsilon}$.

Remark. The previous argument is easily adjusted to yield a new proof of the fact that a direct limit, as in the statement of the proposition, of exact groups is again exact [15]. Indeed, under the assumption of exactness, employing Proposition 2.6 instead of Proposition 2.1, we replace (ii) by

$$
\exists S>0 \text { such that } d_{G_{n}}(g, h) \geq S \Rightarrow\left\langle\xi_{g}, \xi_{h}\right\rangle=0 .
$$

Using (4) and the surrounding discussion, we conclude that this property is shared by $\widehat{\xi}$.

\section{Extensions}

Let $1 \rightarrow H \rightarrow \Gamma \rightarrow G \rightarrow 1$ be an extension of countable discrete groups. We study uniform embeddability of $\Gamma$ under various hypotheses on $H$ and $G$. Our primary result, of which our other results are consequences, is the following theorem.

Theorem 4.1. Let $\Gamma$ be an extension of $H$ by $G$ as above. If $H$ is uniformly embeddable and $G$ is exact, then $\Gamma$ is uniformly embeddable.

As corollaries we mention the following two results about semi-direct products. 
Corollary 4.2. Let $G$ and $H$ be countable discrete groups. Let $\alpha: G \rightarrow \operatorname{Aut}(H)$ be an action of $G$ on $H$. If both $H$ and $G$ are uniformly embeddable and $\alpha(G) \subset$ $\operatorname{Aut}(H)$ is exact, then the semi-direct product $\Gamma=H \rtimes G$ is uniformly embeddable.

Proof. The semi-direct product $\Gamma$ is the set of pairs $(s, x) \in H \times G$, with product $(s, x)(t, y)=\left(s \alpha_{x}(t), x y\right)$. The assignment

$$
(s, x) \mapsto\left(\left(s, \alpha_{x}\right), x\right): \Gamma \rightarrow(H \rtimes \alpha(G)) \times G
$$

defines an injective homomorphism.

By the theorem the semi-direct product $H \rtimes \alpha(G)$ is uniformly embeddable, as is $(H \rtimes \alpha(G)) \times G$. In particular, $\Gamma$ is a subgroup of the uniformly embeddable group and is therefore uniformly embeddable.

Corollary 4.3. Let $\Gamma=\mathbb{Z}^{n} \rtimes G$ be a semi-direct product. If $G$ is uniformly embeddable, then $\Gamma$ is uniformly embeddable.

Proof. Apply the previous corollary, using the fact that $G L_{n}(\mathbb{Z})$ is exact [15].

Remark. Central extensions are more difficult to analyze than semi-direct products. Our theorem applies to central extensions in which the quotient is exact. Gersten has shown that if a central extension of $\mathbb{Z}$ is described by a bounded cocycle, then $\Gamma$ is quasi-isometric to the product $\mathbb{Z} \times G$ 11]. Consequently, if $G$ is uniformly embeddable, so is $\Gamma$. Beyond these two results, little is known.

As remarked earlier, the property of uniform embeddability of a countable discrete group is independent of the proper length function. Consequently, we are free to choose these for our groups $G, H$ and $\Gamma$ in a convenient manner, which we do as follows. Let $l_{\Gamma}$ be a proper length function on $\Gamma$. Define length functions on $H$ and $G$ according to

$$
\begin{aligned}
& l_{H}(s)=l_{\Gamma}(s), \quad \text { for all } s \in H, \\
& l_{G}(x)=\min \left\{l_{\Gamma}(a): a \in \Gamma \text { and } \dot{a}=x\right\}, \quad \text { for all } x \in G,
\end{aligned}
$$

where we have introduced the notation $a \mapsto \dot{a}$ for the quotient map $\Gamma \rightarrow G$. It is easily verified that the minimum in the definition of $l_{G}$ is attained, and that $l_{G}$ is a proper length function on $G$; that $l_{H}$ is a proper length function is immediate. Denote the associated left-invariant metrics by $d_{\Gamma}, d_{G}$ and $d_{H}$, respectively. Observe that the inclusion $H \hookrightarrow \Gamma$ is an isometry, and the quotient map $\Gamma \rightarrow G$ is contractive. Finally, choose a set-theoretic section $\sigma$ of the quotient map $\Gamma \rightarrow G$ with the property that

$$
l_{\Gamma}(\sigma(x))=l_{G}(x), \quad \text { for all } x \in G,
$$

and define $\eta: \Gamma \times G \rightarrow H$ by

$$
\eta(a, x)=\sigma(x)^{-1} a \sigma\left(\dot{a}^{-1} x\right), \quad \text { for all } a \in \Gamma, x \in G .
$$

Lemma 4.4. Let $a, b \in \Gamma, x \in G$. We have

$$
\begin{aligned}
d_{\Gamma}(a, b) & \leq d_{G}(x, \dot{a})+d_{G}(x, \dot{b})+d_{H}(\eta(a, x), \eta(b, x)), \\
d_{H}(\eta(a, x), \eta(b, x)) & \leq d_{G}(x, \dot{a})+d_{G}(x, \dot{b})+d_{\Gamma}(a, b) .
\end{aligned}
$$

Proof. Let $a, b$ and $x \in G$ be as in the statement. From the definition (8) of $\eta$ we obtain

$$
\eta(a, x)^{-1} \eta(b, x)=\sigma\left(\dot{a}^{-1} x\right)^{-1} a^{-1} b \sigma\left(\dot{b}^{-1} x\right) .
$$


The desired inequalities follow easily from this equality, together with (5), (7) and the subadditivity of length functions.

Proof of Theorem 4.1. We prove that $\Gamma$ satisfies the conditions of Proposition 2.1 Let $\varepsilon>0$ and $R>0$ be given. We show that there exists an $f: \Gamma \rightarrow l^{2}(G, \mathcal{H})$ such that $\|f(a)\|=1$ for all $a \in \Gamma$, and that $f$ satisfies the convergence and support properties:

(i) if $d_{\Gamma}(a, b) \leq R$, then $|1-\langle f(a), f(b)\rangle|<\varepsilon$;

(ii) $\forall \hat{\varepsilon}>0 \exists S>0$ such that if $d_{\Gamma}(a, b) \geq S$, then $|\langle f(a), f(b)\rangle|<\hat{\varepsilon}$.

Adapting an argument of Anantharaman-Delaroche and Renault [3], our strategy is to use a $g$ satisfying the conditions of Proposition 2.6 to average an $h$ satisfying those of Proposition 2.1 to produce $f$. Since $G$ is exact, there exist, according to Proposition 2.6. a $g: G \rightarrow l^{2}(G)$ and an $S_{G}>0$ such that $\|g(x)\|=1$ for all $x \in G$ and such that

$\left(\mathrm{i}_{g}\right)|1-\langle g(x), g(y)\rangle|<\varepsilon / 2$, provided $d_{G}(x, y) \leq R ;$

(ii $g) \operatorname{supp} g(x) \subset B_{S_{G}}(x)$, for all $x \in G$.

We shall without comment view $g$ as a function on $G \times G$ whenever convenient. Since $H$ is uniformly embeddable, there exists, according to Proposition 2.1 an $h: H \rightarrow \mathcal{H}$ such that $\|h(s)\|=1$ for all $s \in H$ and such that

$\left(\mathrm{i}_{h}\right)|1-\langle h(s), h(t)\rangle|<\varepsilon / 2$, provided $d_{H}(s, t) \leq 2 S_{G}+R$;

(ii $\left.{ }_{h}\right) \forall \hat{\varepsilon}>0 \exists S_{H}>0$ such that if $d_{H}(s, t) \geq S_{H}$, then $|\langle h(s), h(t)\rangle|<\hat{\varepsilon}$.

Having chosen $g$ and $h$, define $f: \Gamma \rightarrow l^{2}(G, \mathcal{H})$ by

$$
f(a)(x)=g(\dot{a}, x) h(\eta(a, x)), \quad \text { for all } a \in \Gamma, x \in G .
$$

Note that $f(a) \in l^{2}(G, \mathcal{H})$. It is elementary to verify that $\|f(a)\|=1$, for all $a \in \Gamma$. We verify the remaining properties.

For the convergence property, let $a, b \in \Gamma$ with $d_{\Gamma}(a, b) \leq R$. Consider

$$
|1-\langle f(a), f(b)\rangle| \leq\left\{\begin{array}{c}
\mid \sum_{x \in G}\{1-\langle h(\eta(a, x), h(\eta(b, x))\rangle\} g(\dot{a}, x) g(\dot{b}, x) \mid \\
+|1-\langle g(\dot{a}), g(\dot{b})\rangle| .
\end{array}\right.
$$

Since the map $\Gamma \rightarrow G$ is contractive we have $d_{G}(\dot{a}, \dot{b}) \leq R$, so that by $\left(\mathrm{i}_{g}\right)$ the second term in (11) is bounded by $\varepsilon / 2$. Observe that, according to ( $\mathrm{ii}_{g}$ ), the sum in the first term is over $x \in B_{S_{G}}(\dot{a}) \cap B_{S_{G}}(\dot{b})$. Recalling that $\|g(\dot{a})\|=\|g(\dot{b})\|=1$, we therefore bound the first term by

$$
\sup \left\{|1-\langle h(\eta(a, x)), h(\eta(b, x))\rangle|: x \in B_{S_{G}}(\dot{a}) \cap B_{S_{G}}(\dot{b})\right\} .
$$

From (10) we see that for $x \in B_{S_{G}}(\dot{a}) \cap B_{S_{G}}(\dot{b})$ we have $d_{H}(\eta(a, x), \eta(b, x)) \leq$ $2 S_{G}+R$, so that by $\left(\mathrm{i}_{h}\right)$ this supremum, and consequently the first term in (11), is bounded by $\varepsilon / 2$. 
For the support property, let $\hat{\varepsilon}>0$ be given and obtain $S_{H}$ as in $\left(\mathrm{ii}_{h}\right)$. Let $a$, $b \in \Gamma$ be such that $d_{\Gamma}(a, b) \geq 2 S_{G}+S_{H}$. Then,

$$
\begin{aligned}
|\langle f(a), f(b)\rangle| & =\mid \sum_{x \in G} g(\dot{a}, x) g(\dot{b}, x)\langle h(\eta(a, x), h(\eta(b, x))\rangle| \\
& \leq \sum_{x}|g(\dot{a}, x) g(\dot{b}, x)| \mid\langle h(\eta(a, x), h(\eta(b, x))\rangle| \\
& \leq \sup \left\{|\langle h(\eta(a, x)), h(\eta(b, x))\rangle|: x \in B_{S_{G}}(\dot{a}) \cap B_{S_{G}}(\dot{b})\right\},
\end{aligned}
$$

where again we use the fact that $\|g(\dot{a})\|=\|g(\dot{b})\|=1$ to obtain the second inequality, and note that by $\left(\mathrm{ii}_{g}\right)$ the sums are in fact over $x \in B_{S_{G}}(\dot{a}) \cap B_{S_{G}}(\dot{b})$. From (9) we see that for such $x$ we have $d_{H}(\eta(a, x), \eta(b, x)) \geq d_{\Gamma}(a, b)-d_{G}(x, \dot{a})-d_{G}(x, \dot{b}) \geq S_{H}$, so that by $\left(\mathrm{ii}_{h}\right)$ the supremum is indeed bounded by $\hat{\varepsilon}$.

\section{Free Products}

The main result of this section is the following theorem.

Theorem 5.1. Let $A$ and $B$ be countable discrete groups and let $C$ be a common subgroup. If both $A$ and $B$ are uniformly embeddable, then the amalgamated free product $A *_{C} B$ is uniformly embeddable.

Our strategy for proving the theorem is to construct a locally finite metric space $X$ that is uniformly embeddable, and on which $\Gamma$ acts freely by isometries. The construction of $X$ is based on the notion of a tree of metric spaces, which we now recall.

A tree $T$ consists of two sets, a set $V$ of vertices and a set $E$ of edges, together with two endpoint maps $E \rightarrow V$ associating to each edge its endpoints. Every two vertices are connected by a unique geodesic edge path, that is, a path without backtracking.

A tree of spaces $\mathcal{X}$ (with base the tree $T$ ) consists of a family of metric spaces $\left\{X_{v}, X_{e}\right\}$ indexed by the vertices $v \in V$ and edges $e \in E$ of $T$ together with maps $\sigma_{e, v}: X_{e} \rightarrow X_{v}$ whenever $v$ is an endpoint of $e$. The $\sigma_{e, v}$ are the structural maps of $\mathcal{X}$. We will assume, although this is not strictly necessary, that the metrics on the vertex and edge spaces are integer-valued.

The total space $X$ of the tree of spaces $\mathcal{X}$ is the metric space defined as follows. The underlying set of $X$ is the disjoint union of the vertex spaces $X_{v}$; the metric on $X$ is the metric envelope $d$ of the partial metric $\widehat{d}$ (see the appendix) defined by

$$
\widehat{d}(x, y)= \begin{cases}d(x, y), & \text { if } \exists v \in V \text { such that } x, y \in X_{v}, \\ 1, & \text { if } \exists e \in E \text { and } z \in X_{e} \text { such that } x=\sigma_{e, v}(z), y=\sigma_{e, w}(z),\end{cases}
$$

for all $(x, y)$ in the domain

$$
\mathcal{D}=\left\{(x, y): x, y \in X_{v}, v \in V\right\} \cup\left\{\left(\sigma_{e, v}(z), \sigma_{e, w}(z)\right): v \neq w, z \in X_{e}, e \in E\right\} .
$$

Observe that $\mathcal{D}$ is ample (see the appendix); this follows from our assumption that the underlying tree is connected, and that $\widehat{d}$ is defined for all pairs $(x, y)$ where $x$ and $y$ are in the same vertex space.

We call $(x, y)$ an adjacency if there exist an edge $e$, with endpoints $v$ and $w$, and an element $z \in X_{e}$, such that $\sigma_{e, v}(z)=x$ and $\sigma_{e, w}(z)=y$. Using this terminology, 
the partial metric can be described as being the given metric on each vertex space and 1 on adjacencies.

Example 5.2. Consider the case in which the vertex spaces are metric graphs (or rather the set of vertices of a graph, equipped with the graph metric) and the edge spaces are singletons. The total space $X$ is itself a metric graph. Indeed, it is the disjoint union of the graphs, together with additional edges coming from the underlying tree. Precisely, the edges of $X$ are, first, the edges in the individual $X_{v}$, and second, the edges $e$ of the underlying tree; the endpoints of such an edge $e$ are the images of the maps $X_{e} \rightarrow X_{v}$ to the endpoints $v$ of $e$ in $T$.

Remark. The generality of the definition above is mandated by the fact that when considering amalgamated free products, we will encounter vertex spaces that are not metric graphs.

Theorem 5.3. Let $\mathcal{X}$ be a tree of metric spaces in which the structural maps are isometries. If the vertex spaces $X_{v}$ are uniformly embeddable in a Hilbert space (with common distortion bound), then the total space $X$ is uniformly embeddable in a Hilbert space.

Remark. Quite explicitly, the hypothesis is that there exist maps $\rho_{ \pm}$as in (10) such that for every vertex $v \in V$ there exists $F_{v}: X_{v} \rightarrow \mathcal{H}$ satisfying

$$
\rho_{-}\left(d_{X_{v}}\left(x, x^{\prime}\right)\right) \leq\left\|F_{v}(x)-F_{v}\left(x^{\prime}\right)\right\| \leq \rho_{+}\left(d_{X_{v}}\left(x, x^{\prime}\right)\right), \quad \text { for all } x, x^{\prime} \in X_{v} .
$$

In other words, the same distortion bounds may be used for every vertex space.

Remark. If there exist only finitely many distinct isometry types of vertex spaces (as will be the case for the amalgamated free product), this simply means that every vertex space is uniformly embeddable.

Remark. When we prove the theorem we will use the fact that the existence of a common distortion bound on the uniform embeddings of the vertex spaces implies that the estimates in the fundamental criterion for embeddability are uniform. This follows from the proof of Proposition 2.1] specifically from the estimate (2).

Assuming Theorem 5.3 we prepare for the proof Theorem 5.1 by associating a tree of metric spaces $\mathcal{X}_{\Gamma}$ to the amalgamated free product $\Gamma=A *_{C} B$. The tree $T$ is the Bass-Serre tree of $\Gamma$ [17, 4]. Precisely, the vertex and edge sets of $T$ are given by

$$
\begin{aligned}
V & =\Gamma / A \cup \Gamma / B, \\
E & =\Gamma / C,
\end{aligned}
$$

respectively; the endpoint maps are the quotient maps $\Gamma / C \rightarrow \Gamma / A$ and $\Gamma / C \rightarrow$ $\Gamma / B$. In other words, the endpoints of an edge (a $C$-coset) are the vertices (one $A$-coset and one $B$-coset) that contain it.

We associate a metric space to each vertex and edge of $T$ as follows. Equip $\Gamma$ with an integer-valued proper length function and associated metric. Let $v \in V$ be a vertex and assume that $v \in \Gamma / A$. In particular, $v$ is an $A$-coset in $\Gamma$; denote by $X_{v}$ this coset itself, metrized as a subspace of $\Gamma$. Proceed similarly for vertices $v \in \Gamma / B$. Let $e \in E$ be an edge. In particular, $e$ is a $C$-coset in $\Gamma$; denote by $X_{e}$ this coset itself, again metrized as a subspace of $\Gamma$. 
The structural maps are defined as follows. Let the vertex $v$ be an endpoint of the edge $e$. Inclusion of cosets (subsets of $\Gamma$ ) provides the structural map $\sigma_{e, v}$ : $X_{e} \rightarrow X_{v}$.

Remark. The metric space $X_{v}$ is not (isometric to) the graph metric space of the Cayley graph of $A$ or $B$, or even of the restriction of the Cayley graph of $\Gamma$ to the subset $A$ or $B$. Similar remarks apply to the edge space $X_{e}$. Indeed, it is important that we metrize $X_{v}$ and $X_{e}$ as subspaces of $\Gamma$, and not via their identification with $A$ or $B$ and $C$ using the given metrics on these groups.

Let $X_{\Gamma}$ be the total space of $\mathcal{X}_{\Gamma}$. The group $\Gamma$ acts on $X_{\Gamma}$ by left multiplication. Precisely, for $x \in X_{v}$ and $a \in \Gamma$ we define $a \cdot x \in X_{a \cdot v}$, using ordinary multiplication in $\Gamma$, by virtue of the fact that $x \in v \subset \Gamma$. This action preserves adjacencies and, since in addition the metric on $\Gamma$ is left-invariant, it preserves the partial metric. According to Proposition 7.2 of the appendix, $\Gamma$ acts by isometries on the total space $X_{\Gamma}$.

Proposition 5.4. Let $A$ and $B$ be countable discrete groups and let $C$ be a common subgroup. Let $\Gamma=A *_{C} B$ be the amalgamated free product. Let $\mathcal{X}_{\Gamma}$ be the tree of metric spaces associated to $\Gamma$ and let $X_{\Gamma}$ be its total space. We have:

(i) The structural maps of $\mathcal{X}_{\Gamma}$ are isometries.

(ii) Every vertex space of $\mathcal{X}_{\Gamma}$ is isometric to one of $A$ or $B$ (which are metrized with the subspace metric via the inclusions $A, B \subset \Gamma$ ).

(iii) The action of $\Gamma$ by isometries on $X_{\Gamma}$ is free.

(iv) $X_{\Gamma}$ is locally finite.

Proof. All of the assertions but (iv) follow from the previous discussion. The condition (iv), while apparent, will be derived formally in Proposition 5.6.

Proof of Theorem 5.1. According to the previous proposition, the tree of metric spaces $\mathcal{X}_{\Gamma}$ associated to the amalgamated free product $\Gamma=A *_{C} B$ satisfies the hypothesis of Theorem 5.3. according to which the total space $X_{\Gamma}$ is uniformly embeddable. Again according to the previous proposition, $\Gamma$ acts freely by isometries on $X_{\Gamma}$. Hence, by Corollary 2.5, $\Gamma$ is uniformly embeddable.

We have reduced our main theorem concerning amalgamated free products, Theorem 5.1, to a theorem concerning trees of metric spaces, Theorem 5.3. We now turn attention to the proof of that theorem. We suitably adapt the method employed by $\mathrm{Tu}$ in his study of Property A for discrete metric spaces [19].

For a tree of metric spaces $\mathcal{X}$ the inclusions $X_{v} \rightarrow X$ of vertex spaces into the total space are, as a general rule, not isometric; the presence of "shortcuts" in neighboring $X_{w}$ may cause the distance in $X$ between two vertices $x, y \in X_{v}$ to be considerably smaller than the distance between them in $X_{v}$ itself. Nevertheless, for our $\mathcal{X}_{\Gamma}$ these inclusions are isometries, a fact that may be traced back to the manner in which the vertex and edge spaces of $\mathcal{X}_{\Gamma}$ are metrized as subspaces of $\Gamma$, which circumvents any distortion that may have otherwise been introduced by the amalgamating subgroup. The following proposition has no analog in Tu's work 19 ; nevertheless, we require it in order to complete our arguments.

Proposition 5.5. Let $\mathcal{X}=\left\{X_{v}, X_{e}\right\}$ be a tree of metric spaces in which the structural maps $X_{e} \rightarrow X_{v}$ are isometries. Let $X$ be the total space of $\mathcal{X}$. Then

(i) the inclusions $X_{v} \rightarrow X$ are isometries, and 
(ii) if $(x, y)$ is an adjacency, then $d(x, y)=1$.

Further, for all $x \in X_{v}, y \in X_{w}$,

$$
d(x, y)=d_{T}(v, w)+\inf \left\{d\left(x_{0}, x_{1}\right)+d\left(x_{2}, x_{3}\right)+\cdots+d\left(x_{p-1}, x_{p}\right)\right\},
$$

where $d_{T}$ is the distance on the tree $T$ and the infimum is taken over all sequences $x_{0}, \ldots, x_{p}$, where $p=2 d_{T}(v, w)+1$, and

(i) $x=x_{0}, y=x_{p}$,

(ii) $\left(x_{2 k-1}, x_{2 k}\right)$ is an adjacency for $k=1, \ldots, d_{T}(v, w)$,

(iii) $x_{2 k}, x_{2 k+1} \in X_{v_{k}}$, for $k=0, \ldots, d_{T}(v, w)$, and

$v=v_{0}, \ldots, v_{d_{T}(v, w)}=w$ are the vertices along the unique geodesic path in $T$ from $v$ to $w$.

Proof. Let $v, w \in V$ and let $x \in X_{v}$ and $y \in X_{w}$. A reduced path from $x$ to $y$ is a sequence of elements $x=x_{0}, x_{1}, \ldots, x_{2 n}, x_{2 n+1}$ of $X$ for which there exist vertices $v_{0}, \ldots, v_{n} \in V$ such that

(i) $v_{j} \neq v_{j+1}$, for $j=1, \ldots, n$, and

(ii) $x_{2 k}, x_{2 k+1} \in X_{v_{k}}$, for $k=0,1, \ldots, n$.

Observe that by appropriately inserting and deleting $x$ 's we may alter a path, without increasing its length, so as to obtain a reduced path (use the triangle inequality for the metrics on the individual $X_{v}$ ). Consequently, when computing distances in $X$ it suffices to consider reduced paths.

Let $x=x_{0}, \ldots, x_{2 n+1}=y$ be a reduced path from $x$ to $y$. According to the definitions of a reduced path and of the domain $\mathcal{D}$ of the partial metric we obtain sequences of

(i) vertices $v=v_{0}, \ldots, v_{n}=w$ in $V$,

(ii) edges $e_{1}, \ldots, e_{n}$ in $E$, and

(iii) elements $z_{1}, \ldots, z_{n}$ of the edge spaces $X_{e_{1}}, \ldots, X_{e_{n}}$, satisfying

(i) $x_{2 k}, x_{2 k+1} \in X_{v_{k}}$, for $k=0, \ldots, n$,

(ii) the endpoints of $e_{k}$ are $v_{k-1}$ and $v_{k}$, for $k=1, \ldots, n$, and

(iii) $\sigma_{e_{k}, v_{k-1}}\left(z_{k}\right)=x_{2 k-1}$ and $\sigma_{e_{k}, v_{k}}\left(z_{k}\right)=x_{2 k}$, for $k=1, \ldots, n$.

(The sequences are uniquely determined by these conditions.) The given reduced path $x=x_{0}, \ldots, x_{p}=y$ in $X$ lies over the edge path $e_{1}, \ldots, e_{n}$ in $T$.

We show that in the definition of $d$ it suffices to consider reduced paths lying over the unique geodesic edge path in $T$ from $v$ to $w$; the assertions of the proposition follow easily from this fact. Let $x=x_{0}, \ldots, x_{2 n+1}=y$ lie over the non-geodesic path $e_{1}, \ldots, e_{n}$ in $T$. We show that by successive elimination of certain $x_{i}$ we obtain a shorter path lying over the geodesic path in $T$. Indeed, there exists $i \in 1, \ldots, n-1$ such that $e_{i}$ and $e_{i+1}$ have the same endpoints, that is, such that $v_{i-1}=v_{i+1}$. We have

$$
\left.\left.\left.\begin{array}{l}
x_{2 i-2} \\
x_{2 i-1}
\end{array}\right\} \in X_{v_{i-1}}, \quad \begin{array}{r}
x_{2 i} \\
x_{2 i+1}
\end{array}\right\} \in X_{v_{i}}, \quad \begin{array}{l}
x_{2 i+2} \\
x_{2 i+3}
\end{array}\right\} \in X_{v_{i+1}}=X_{v_{i-1}} .
$$

We eliminate $x_{2 i-1}, x_{2 i}, x_{2 i+1}, x_{2 i+2}$ from the given path and claim that the resulting path

(i) is shorter than $x_{0}, \ldots, x_{2 n+1}$, and

(ii) lies over an edge path with fewer backtracks than $e_{1}, \ldots, e_{n}$. 
Of these, (ii) is obvious; the new path lies over the edge path $e_{1}, \ldots, e_{i-1}, e_{i+2}, \ldots$, $e_{n}$ obtained by eliminating $e_{i}$ and $e_{i+1}$. Further, (i) follows from the fact that the structural maps are isometries. In particular, we have

$$
d\left(x_{2 i-1}, x_{2 i+2}\right)=d\left(z_{i}, z_{i+1}\right)=d\left(x_{2 i}, x_{2 i+1}\right),
$$

from which follows

$$
\begin{aligned}
d\left(x_{2 i-2}, x_{2 i+3}\right) & \leq d\left(x_{2 i-2}, x_{2 i-1}\right)+d\left(x_{2 i-1}, x_{2 i+2}\right)+d\left(x_{2 i+2}, x_{2 i+3}\right) \\
& =d\left(x_{2 i-2}, x_{2 i-1}\right)+d\left(x_{2 i}, x_{2 i+1}\right)+d\left(x_{2 i+2}, x_{2 i+3}\right) .
\end{aligned}
$$

Proposition 5.6. Let $X$ be the total space of a tree of metric spaces $\mathcal{X}$ in which the structural maps are isometries. Assume that each vertex space $X_{v}$ is locally finite and there is a uniform bound for the number of adjacencies of each point in $X$. Then $X$ is locally finite.

Proof. In view of formula (12), a finite radius ball in $X$ can intersect only finitely many vertex spaces.

\section{Proof of Theorem 5.3}

The main result of this section is Proposition 6.8 which implies Theorem 5.3 and at the same time reproves the exactness of free products with amalgam of exact groups.

We may enlarge the tree of metric spaces (if necessary) so that the underlying tree $T$ will contain an infinite geodesic $\omega$ starting at some basepoint. For every $v \in V$ let $\alpha(v) \in V$ be such that the edge $[v, \alpha(v)]$ points towards $\omega$. For each $v \in V$ let $Y_{v}=\sigma_{e, v}\left(X_{e}\right) \subset X_{v}$ and $f_{v}=\sigma_{e, \alpha(v)} \circ \sigma_{e, v}^{-1}: Y_{v} \rightarrow X_{\alpha(v)}$ where $e=[v, \alpha(v)]$. It follows immediately from Proposition [5.5 that each $f_{v}$ is isometric.

Using the same proposition, and the notation just introduced, we rewrite the distance formula (12) as follows. Let $x_{0} \in X_{v}$ and $x_{0}^{\prime} \in X_{v^{\prime}}$. There exists a unique pair of nonnegative integers $k, \ell$ such that $\alpha^{k}(v)=\alpha^{\ell}\left(v^{\prime}\right)$ and $d_{T}\left(v, v^{\prime}\right)=k+\ell$, where again $d_{T}$ denotes the distance in the tree $T$. By symmetry we may assume that $k \geq \ell$. If $k \geq 1$ and $\ell \geq 1$, then

$$
d\left(x_{0}, x_{0}^{\prime}\right)=k+\ell+\inf \left\{\begin{array}{c}
d\left(x_{0}, y_{0}\right)+\sum_{i=0}^{k-2} d\left(f_{\alpha^{i}(v)}\left(y_{i}\right), y_{i+1}\right) \\
+d\left(f_{\alpha^{k-1}(v)}\left(y_{k-1}\right), f_{\alpha^{\ell-1}\left(v^{\prime}\right)}\left(y_{\ell-1}^{\prime}\right)\right) \\
\quad+\sum_{j=0}^{\ell-2} d\left(f_{\alpha^{j}\left(v^{\prime}\right)}\left(y_{j}^{\prime}\right), y_{j+1}^{\prime}\right)+d\left(y_{0}^{\prime}, x_{0}^{\prime}\right)
\end{array}\right\},
$$

subject to the constraints that $y_{i} \in Y_{\alpha^{i}(v)}$ and $y_{j}^{\prime} \in Y_{\alpha^{j}\left(v^{\prime}\right)}$. If $k \geq 1$ and $\ell=0$, then

$$
\left.d\left(x_{0}, x_{0}^{\prime}\right)=k+\inf \left\{d\left(x_{0}, y_{0}\right)+\sum_{i=0}^{k-2} d\left(f_{\alpha^{i}(v)}\left(y_{i}\right), y_{i+1}\right)+d\left(f_{\alpha^{k-1}(v)}\left(y_{k-1}\right), x_{0}^{\prime}\right)\right)\right\},
$$

subject to similar constraints.

Remark. Formulas (13) and (14) are the same as Tu's formulas [19, Section 9]. 
An $n$-chain is a sequence $\mathbf{x}=\left(x_{0}, x_{1}, \ldots, x_{n-1}\right)$ with $x_{k} \in X_{\alpha^{k}(v)}$ such that for each $0 \leq k \leq n-2$ there exists $\bar{x}_{k} \in Y_{\alpha^{k}(v)}$ satisfying $d\left(x_{k}, \bar{x}_{k}\right)<d\left(x_{k}, Y_{\alpha^{k}(v)}\right)+1$ and $f_{\alpha^{k}(v)}\left(\bar{x}_{k}\right)=x_{k+1}$. If $x_{0} \in X_{v}$, the $n$-chain starts in $X_{v}$. Note that for any $n \geq 1, v \in V$ and $x_{0} \in X_{v}$ there exists an $n$-chain whose initial element is $x_{0}$.

Lemma 6.1. Let $x_{0} \in X_{v}$ and $x_{0}^{\prime} \in X_{v^{\prime}}$ with $d\left(x_{0}, x_{0}^{\prime}\right)<R$, and let $k$ and $\ell$ be as in (13) and (14). Then there exist chains $\left(x_{0}, x_{1}, \ldots, x_{k}\right),\left(x_{0}^{\prime}, x_{1}^{\prime}, \ldots, x_{\ell}^{\prime}\right)$ such that

$$
\max \left\{\left(\sup _{0 \leq i \leq k-1} d\left(x_{i}, x_{i+1}\right)\right),\left(\sup _{0 \leq j \leq \ell-1} d\left(x_{j}^{\prime}, x_{j+1}^{\prime}\right)\right), d\left(x_{k}, x_{\ell}^{\prime}\right)\right\}<2^{R} R .
$$

Remark. In the proof of the lemma, and at a number of subsequent points, we require the fact that if $x \in X_{v}$ and $y \in Y_{v}$, then

$$
d\left(x, f_{v}(y)\right)=d(x, y)+1 .
$$

This follows from Theorem 5.5. Indeed, since $\left(y, f_{v}(y)\right)$ is an adjacency, $d\left(x, f_{v}(y)\right)$ $\leq d(x, y)+1$. For the reverse inequality, let $\varepsilon>0$ and obtain $x^{\prime} \in Y_{v}$ such that $\bar{d}\left(x, f_{v}(y)\right)+\varepsilon \geq 1+d\left(x, x^{\prime}\right)+d\left(f_{v}\left(x^{\prime}\right), f_{v}(y)\right)$. Since $f_{v}$ is an isometry, we get $d\left(x, x^{\prime}\right)+d\left(f_{v}\left(x^{\prime}\right), f_{v}(y)\right) \geq d(x, y)$, and we are done.

Proof. If $v=v^{\prime}$ there is nothing to prove; so we may assume that $v \neq v^{\prime}$. By symmetry we may also assume that $k \geq \ell$; hence $k \geq 1$ since $v \neq v^{\prime}$.

Case $\ell=0$ : We need to prove that there exists a chain $\left(x_{0}, x_{1}, \ldots, x_{k}\right)$ such that

$$
\max \left\{\left(\sup _{0 \leq i \leq k-1} d\left(x_{i}, x_{i+1}\right)\right), d\left(x_{k}, x_{0}^{\prime}\right)\right\}<2^{k} R \leq 2^{R} R .
$$

Since $d\left(x_{0}, x_{0}^{\prime}\right)<R$, by (14) there is $y_{0} \in Y_{v}$ such that $k+d\left(x_{0}, y_{0}\right)<R$. The sequence $x_{1}, \ldots, x_{k}$ is constructed inductively. Let $\bar{x}_{0} \in Y_{v}$ be such that $d\left(x_{0}, \bar{x}_{0}\right) \leq$ $d\left(x_{0}, y_{0}\right)$ and $d\left(x_{0}, \bar{x}_{0}\right)<d\left(x_{0}, Y_{v}\right)+1$, and define $x_{1}=f_{v}\left(\bar{x}_{0}\right)$. Then

$$
d\left(x_{0}, x_{1}\right)=d\left(x_{0}, \bar{x}_{0}\right)+1 \leq d\left(x_{0}, y_{0}\right)+1<R .
$$

Thus $d\left(x_{0}, x_{1}\right)<R$ and $d\left(x_{1}, x_{0}^{\prime}\right) \leq d\left(x_{0}, x_{0}^{\prime}\right)+d\left(x_{0}, x_{1}\right)<2 R$. Repeating the same argument for the pair of points $x_{1}, x_{0}^{\prime}$ (if $k \geq 2$ ), we find $x_{2} \in X_{\alpha^{2}(v)}$ with $\left(x_{0}, x_{1}, x_{2}\right)$ being a chain, $d\left(x_{1}, x_{2}\right)<2 R$ and $d\left(x_{2}, x_{0}^{\prime}\right)<2^{2} R$. Continuing in the same way, we obtain a chain $\left(x_{0}, x_{1}, \ldots, x_{k}\right)$ such that $d\left(x_{i-1}, x_{i}\right)<2^{i-1} R$ and $d\left(x_{i}, x_{0}^{\prime}\right)<2^{i} R, 1 \leq i \leq k$. Since $k \leq R$, this completes the proof for $\ell=0$.

Case $\ell \geq 1$ : Let $y_{0}, \ldots, y_{k-1}$ with $y_{i} \in Y_{\alpha^{i}(v)}$ and $y_{0}^{\prime}, \ldots, y_{\ell-1}^{\prime}$ with $y_{j}^{\prime} \in Y_{\alpha^{j}\left(v^{\prime}\right)}$ be sequences such that the expression whose infimum is taken in (13) is less than $R-k-\ell$. Let $z=f_{\alpha^{k-1}(v)}\left(y_{k-1}\right)$ and $z^{\prime}=f_{\alpha^{\ell-1}\left(v^{\prime}\right)}\left(y_{\ell-1}^{\prime}\right)$. Then we have $d\left(x_{0}, z\right)+$ $d\left(z, z^{\prime}\right)+d\left(x_{0}^{\prime}, z^{\prime}\right)<R$, so that both $d\left(x_{0}, z\right)$ and $d\left(x_{0}^{\prime}, z\right)$ are less than $R$. The proof is completed by applying the first part of the proof to the pairs $x_{0}, z$ and $x_{0}^{\prime}, z$ and noting that

$$
d\left(x_{k}, x_{\ell}^{\prime}\right) \leq d\left(x_{k}, z\right)+d\left(x_{\ell}^{\prime}, z\right)<2^{k} R+2^{\ell} R \leq 2^{k+\ell} R \leq 2^{R} R .
$$

Given an $n$-chain $\mathbf{x}=\left(x_{0}, x_{1}, \ldots, x_{n-1}\right)$ starting in $X_{v}$, define, for $0 \leq k \leq$ $n-1, \delta_{k}=d\left(x_{k}, Y_{\alpha^{k}(v)}\right)$ and $\theta_{k}=\delta_{0} \vee \cdots \vee \delta_{k}$, where we introduce the notation $a \vee b=\max \{a, b\}$. For future notational convenience define $\theta_{-1}=0$. Note that if $\mathbf{x}^{\prime}=\left(x_{0}^{\prime}, x_{1}^{\prime}, \ldots, x_{n-1}^{\prime}\right)$ is another $n$-chain starting in $X_{v}$, then

$$
\begin{gathered}
d\left(x_{k-1}, x_{k}\right) \leq \delta_{k-1}+2, \\
\left|\delta_{k}-\delta_{k}^{\prime}\right|=\left|d\left(x_{k}, Y_{\alpha^{k}(v)}\right)-d\left(x_{k}^{\prime}, Y_{\alpha^{k}(v)}\right)\right| \leq d\left(x_{k}, x_{k}^{\prime}\right), \quad 0 \leq k \leq n-1 .
\end{gathered}
$$


Lemma 6.2. Let $\mathbf{x}=\left(x_{0}, x_{1}, \ldots, x_{n-1}\right)$ and $\mathbf{x}^{\prime}=\left(x_{0}^{\prime}, x_{1}^{\prime}, \ldots, x_{n-1}^{\prime}\right)$ be $n$-chains starting in $X_{v}$. Then

$$
\left|d\left(x_{k}, x_{k}^{\prime}\right)-d\left(x_{0}, x_{0}^{\prime}\right)\right| \leq 2 k\left(\theta_{k-1} \vee \theta_{k-1}^{\prime}\right)+2 k, \quad 0 \leq k \leq n-1 .
$$

Proof. Since the statement is obvious in the case $k=0$, we assume $k \geq 1$. Let $\bar{x}_{k}$ and $\bar{x}_{k}^{\prime}$ be as in the definition of $n$-chains. Observe that

$$
\begin{aligned}
& d\left(\bar{x}_{k}, \bar{x}_{k}^{\prime}\right) \leq d\left(\bar{x}_{k}, x_{k}\right)+d\left(\bar{x}_{k}^{\prime}, x_{k}^{\prime}\right)+d\left(x_{k}, x_{k}^{\prime}\right) \leq d\left(x_{k}, x_{k}^{\prime}\right)+2\left(\theta_{k} \vee \theta_{k}^{\prime}\right)+2, \\
& d\left(\bar{x}_{k}, \bar{x}_{k}^{\prime}\right) \geq d\left(x_{k}, x_{k}^{\prime}\right)-d\left(\bar{x}_{k}, x_{k}\right)-d\left(\bar{x}_{k}^{\prime}, x_{k}^{\prime}\right) \geq d\left(x_{k}, x_{k}^{\prime}\right)-2\left(\theta_{k} \vee \theta_{k}^{\prime}\right)-2 .
\end{aligned}
$$

Since $f_{\alpha^{k}(v)}$ is an isometry we have $d\left(x_{k+1}, x_{k+1}^{\prime}\right)=d\left(\bar{x}_{k}, \bar{x}_{k}^{\prime}\right)$, and the lemma follows from these inequalities by induction.

Given an $n$-chain $\mathbf{x}=\left(x_{0}, x_{1}, \ldots, x_{n-1}\right)$ and $N>0$, we define, for $0 \leq k \leq n-1$,

$$
\begin{aligned}
& a_{k}=\left(1-\frac{\ln \left(1+\theta_{k}\right)}{N}\right)_{+}, \\
& c_{k}=\sqrt{a_{0} \cdots a_{k-1}\left(1+(n-k-1)\left(1-a_{k}\right)\right)},
\end{aligned}
$$

where $a_{+}=\max \{a, 0\}$. Note that $c_{0}=\sqrt{1+(n-1)\left(1-a_{0}\right)}$. One checks immediately that $a_{0} \geq a_{1} \geq \cdots \geq a_{n-1}$,

$$
\begin{gathered}
c_{0}^{2}+\cdots+c_{n-1}^{2}=n, \quad 0 \leq c_{n-1} \leq 1, \\
\delta_{k} \geq e^{N}-1 \Longrightarrow \theta_{k} \geq e^{N}-1 \Longrightarrow a_{k}=0 \Longrightarrow c_{k+1}=\cdots=c_{n-1}=0 .
\end{gathered}
$$

The coefficients $c_{k}$ were introduced by Tu [19] (actually we work with the square root of Tu's coefficients). Since the maps $f_{v}$ are isometries, it is possible in our case to define $a_{k}$ explicitly as in (18). Both $a_{k}$ and $c_{k}$ should be regarded as functions $a_{k}^{\mathbf{x}}$ and $c_{k}^{\mathbf{x}}$ of $n$-chains $\mathbf{x}=\left(x_{0}, x_{1}, \ldots, x_{n-1}\right)$. We will often write $a_{k}^{\prime}$ and $c_{k}^{\prime}$ instead of $a_{k}^{\mathbf{x}^{\prime}}$ and $c_{k}^{\mathbf{x}^{\prime}}$.

Lemma 6.3. Let $\mathbf{x}=\left(x_{0}, x_{1}, \ldots, x_{n-1}\right)$ and $\mathbf{x}^{\prime}=\left(x_{0}^{\prime}, x_{1}^{\prime}, \ldots, x_{n-1}^{\prime}\right)$ be $n$-chains starting in $X_{v}$. If $\omega=\max _{0 \leq i \leq n-1}\left|a_{i}-a_{i}^{\prime}\right|$, then

(i) $\left|c_{k}^{2}-c_{k}^{\prime 2}\right| \leq\left(\frac{n+1}{2}\right)^{2} \omega$,

(ii) $\left|c_{k}-c_{k}^{\prime}\right| \leq \frac{n+1}{\sqrt{2}} \sqrt{\omega}$, and

(iii) $\sum_{k=0}^{n-1} \frac{1}{n}\left|c_{k}-c_{k}^{\prime}\right|^{2} \leq \frac{(n+1)^{2}}{2} \omega$.

Proof. This is an exercise. For (i) $\Rightarrow$ (ii) use the inequality $|\sqrt{a}-\sqrt{b}| \leq \sqrt{2|a-b|}$.

The following continuity property of the coefficients $a_{k}$ is a minor variation of a formula in Tu's paper [19, formula 9.2].

Lemma 6.4. Let $\mathbf{x}=\left(x_{0}, x_{1}, \ldots, x_{n-1}\right)$ and $\mathbf{x}^{\prime}=\left(x_{0}^{\prime}, x_{1}^{\prime}, \ldots, x_{n-1}^{\prime}\right)$ be $n$-chains starting in $X_{v}$, and assume $d\left(x_{0}, x_{0}^{\prime}\right) \leq n$. Then

$$
\max _{0 \leq k \leq n-1}\left|a_{k}-a_{k}^{\prime}\right| \leq \frac{7 n^{2}}{N} \text {. }
$$

Proof. Denote $\lambda=\ln 7 n$. We are going to show that

$$
\begin{aligned}
\left|a_{0}-a_{0}^{\prime}\right| & \leq \frac{n}{N}, \\
\left|a_{k}-a_{k}^{\prime}\right| & \leq\left|a_{k-1}-a_{k-1}^{\prime}\right|+\frac{\lambda}{N} .
\end{aligned}
$$


From these we immediately obtain $\left|a_{k}-a_{k}^{\prime}\right| \leq \frac{n(1+\lambda)}{N} \leq \frac{7 n^{2}}{N}$, concluding the proof. Of these inequalities, the first is straightforward. Indeed, using (16) and the property that the map $t \mapsto\left(1-\frac{1}{N} \ln (1+t)\right)_{+}$is $\frac{1}{N}$-Lipschitz, we have

$$
\left|a_{0}-a_{0}^{\prime}\right| \leq \frac{1}{N}\left|\delta_{0}-\delta_{0}^{\prime}\right| \leq \frac{1}{N} d\left(x_{0}, x_{0}^{\prime}\right) \leq \frac{n}{N} .
$$

To prove the second inequality, denote $f(t)=4 n t+6 n$. According to (16) and Lemma 6.2 we have

$$
\left|\delta_{k}-\delta_{k}^{\prime}\right| \leq d\left(x_{k}, x_{k}^{\prime}\right) \leq \frac{1}{2} f\left(\theta_{k-1} \vee \theta_{k-1}^{\prime}\right) .
$$

Denote $\psi(t)=\ln (1+t)$, so that for all $t \geq 0$ we have

$$
\psi(t) \leq \psi(f(t)) \leq \psi(t)+\lambda .
$$

Case $\theta_{k} \vee \theta_{k}^{\prime} \leq f\left(\theta_{k-1} \vee \theta_{k-1}^{\prime}\right)$ : By symmetry we may assume that $\theta_{k-1} \geq \theta_{k-1}^{\prime}$. Thus $\theta_{k-1}^{\prime} \leq \theta_{k}^{\prime} \leq f\left(\theta_{k-1}\right)$ and $\theta_{k-1}^{\prime} \leq \theta_{k-1} \leq \theta_{k} \leq f\left(\theta_{k-1}\right)$. Using (25), we obtain

$$
\begin{aligned}
& \psi\left(\theta_{k-1}^{\prime}\right) \leq \psi\left(\theta_{k}^{\prime}\right) \leq \psi\left(f\left(\theta_{k-1}\right)\right) \leq \psi\left(\theta_{k-1}\right)+\lambda, \\
& \psi\left(\theta_{k-1}^{\prime}\right) \leq \psi\left(\theta_{k}\right) \leq \psi\left(f\left(\theta_{k-1}\right)\right) \leq \psi\left(\theta_{k-1}\right)+\lambda .
\end{aligned}
$$

Now, (23) follows immediately from these inequalities, together with the property that for real numbers $a \leq s, t \leq b+\lambda$ we have

$$
\left|(1-s / N)_{+}-(1-t / N)_{+}\right| \leq\left|(1-b / N)_{+}-(1-a / N)_{+}\right|+\lambda / N .
$$

Case $\theta_{k} \vee \theta_{k}^{\prime} \geq f\left(\theta_{k-1} \vee \theta_{k-1}^{\prime}\right):$ By symmetry we may assume that $\theta_{k} \geq \theta_{k}^{\prime}$. Then $\overline{\theta_{k} \geq f\left(\theta_{k-1}\right) \geq \theta_{k-1}, \text { and hence }} \theta_{k}=\delta_{k}$. Therefore, using (24),

$$
\theta_{k} \leq\left|\theta_{k}-\theta_{k}^{\prime}\right|+\theta_{k}^{\prime} \leq\left|\delta_{k}-\delta_{k}^{\prime}\right|+\theta_{k}^{\prime} \leq \frac{1}{2} f\left(\theta_{k-1} \vee \theta_{k-1}^{\prime}\right)+\theta_{k}^{\prime} \leq \frac{1}{2} \theta_{k}+\theta_{k}^{\prime} .
$$

Hence $\theta_{k}^{\prime} \leq \theta_{k} \leq 2 \theta_{k}^{\prime}$. We obtain

$$
\psi\left(\theta_{k}^{\prime}\right) \leq \psi\left(\theta_{k}\right) \leq \psi\left(\theta_{k}^{\prime}\right)+\ln 2 .
$$

From this inequality and the property that the map $t \mapsto(1-t / N)_{+}$is $\frac{1}{N}$-Lipschitz, we obtain $\left|a_{k}-a_{k}^{\prime}\right| \leq \frac{\ln 2}{N} \leq \frac{\lambda}{N}$, which implies (23).

Definition. Given $R>0$ and $\varepsilon>0$ choose and fix $n \in \mathbb{N}$ such that

$$
\left(\frac{\ln n+9}{4 n}\right)^{\frac{1}{2}}<\frac{\varepsilon}{3(R+1)}, \quad n>2^{R} R,
$$

and $N \in \mathbb{N}$ such that

$$
\frac{6 n(n+1)}{\sqrt{N}}<\frac{\varepsilon}{3(R+1)} .
$$

Having done so, apply the fundamental criterion for uniform embeddability, Proposition 2.1 to choose and fix a family $\left(\xi_{x}\right)_{x \in X}$ of unit vectors in a Hilbert space $\mathcal{H}_{X}=\bigoplus_{v \in V} \mathcal{H}_{v}$ with $\xi_{x} \in \mathcal{H}_{v}$ if $x \in X_{v}$ and such that

$$
\begin{gathered}
\sup \left\{\left\|\xi_{y}-\xi_{y^{\prime}}\right\|: d\left(y, y^{\prime}\right) \leq 3 n e^{N}, y, y^{\prime} \in X_{v}, v \in V\right\}<\frac{\varepsilon}{3(R+1)}, \\
\lim _{S \rightarrow \infty} \sup \left\{\left|\left\langle\xi_{y}, \xi_{y^{\prime}}\right\rangle\right|: d\left(y, y^{\prime}\right) \geq S, y, y^{\prime} \in X_{v}, v \in V\right\}=0 .
\end{gathered}
$$


(See the remarks after Theorem 5.3 for comments on why this is possible.) Finally, for every $n$-chain $\mathbf{x}=\left(x_{0}, x_{1}, \ldots, x_{n-1}\right)$ in $X$ define the unit vector $\eta^{\mathbf{x}} \in \mathcal{H}_{X}$ by

$$
\eta^{\mathbf{x}}=\frac{1}{\sqrt{n}} \sum_{k=0}^{n-1} c_{k} \xi_{x_{k}}
$$

where, of course, the $c_{k}$ 's are defined according to (19) and depend on the chain $\mathbf{x}$.

Lemma 6.5. Let $\mathbf{x}=\left(x_{0}, x_{1}, \ldots, x_{n-1}\right)$ and $\mathbf{x}^{\prime}=\left(x_{0}^{\prime}, x_{1}^{\prime}, \ldots, x_{n-1}^{\prime}\right)$ be $n$-chains starting in $X_{v}$. If $d\left(x_{0}, x_{0}^{\prime}\right) \leq n$, then $\left\|\eta^{\mathbf{x}}-\eta^{\mathbf{x}^{\prime}}\right\| \leq \frac{2 \varepsilon}{3(R+1)}$.

Proof. Let $I=\left\{k: c_{k} \neq 0, c_{k}^{\prime} \neq 0\right\}$ and $J=\left\{k: c_{k}=0, c_{k}^{\prime} \neq 0\right\}$. If $1 \leq k \in I$, then $a_{k-1}=\left(1-\frac{1}{N} \ln \left(1+\theta_{k-1}\right)\right)_{+} \neq 0$; hence $\theta_{k-1} \leq e^{N}-1$. Similarly, $\theta_{k-1}^{\prime} \leq e^{N}-1$. Hence from (17) we have

$$
d\left(x_{k}, x_{k}^{\prime}\right) \leq 2 n\left(e^{N}-1\right)+2 n+d\left(x_{0}, x_{0}^{\prime}\right) \leq 3 n e^{N} .
$$

Consider

$$
\begin{aligned}
\left\|\eta^{\mathbf{x}}-\eta^{\mathbf{x}^{\prime}}\right\|= & \left\|\frac{1}{\sqrt{n}} \sum_{k=0}^{n-1} c_{k} \xi_{x_{k}}-\frac{1}{\sqrt{n}} \sum_{k=0}^{n-1} c_{k}^{\prime} \xi_{x_{k}^{\prime}}\right\| \\
\leq & \left\{\begin{aligned}
\left\|\frac{1}{\sqrt{n}} \sum_{k=0}^{n-1}\left(c_{k}-c_{k}^{\prime}\right) \xi_{x_{k}}\right\| & +\left\|\frac{1}{\sqrt{n}} \sum_{k \in J}\left(c_{k}^{\prime}-c_{k}\right)\left(\xi_{x_{k}}-\xi_{x_{k}^{\prime}}\right)\right\| \\
+ & \left\|\frac{1}{\sqrt{n}} \sum_{k \in I} c_{k}^{\prime}\left(\xi_{x_{k}}-\xi_{x_{k}^{\prime}}\right)\right\| .
\end{aligned}\right.
\end{aligned}
$$

Observe that the $\xi_{x_{k}}$ 's and $\xi_{x_{k}^{\prime}}$ 's are in orthogonal components of $\mathcal{H}_{X}$. We bound the third term on the right using (20), (31) and (28) as follows:

$$
\begin{aligned}
\frac{1}{\sqrt{n}}\left(\sum_{k \in I}\left(c_{k}^{\prime}\right)^{2}\left\|\xi_{x_{k}}-\xi_{x_{k}^{\prime}}\right\|^{2}\right)^{1 / 2} & \leq \frac{1}{\sqrt{n}}\left(\sum_{k=0}^{n-1}\left(c_{k}^{\prime}\right)^{2}\right)^{1 / 2} \cdot\left(\sup _{k \in I}\left\|\xi_{x_{k}}-\xi_{x_{k}^{\prime}}\right\|\right) \\
& \leq \sup _{\Delta}\left\|\xi_{y}-\xi_{y^{\prime}}\right\| \leq \frac{\varepsilon}{3(R+1)},
\end{aligned}
$$

where $\Delta=\left\{\left(y, y^{\prime}\right): d\left(y, y^{\prime}\right) \leq 3 n e^{N}, y, y^{\prime} \in X_{v}, v \in V\right\}$. We bound the sum of the first two terms on the right, by

$$
\begin{aligned}
3\left(\sum_{k=0}^{n-1} \frac{1}{n}\left|c_{k}-c_{k}^{\prime}\right|^{2}\right)^{\frac{1}{2}} & \leq \frac{3(n+1)}{\sqrt{2}} \max _{0 \leq i \leq n-1}\left|a_{i}-a_{i}^{\prime}\right|^{\frac{1}{2}} \\
& \leq \frac{3(n+1)}{\sqrt{2}}\left(\frac{7 n^{2}}{N}\right)^{\frac{1}{2}} \leq \frac{\varepsilon}{3(R+1)},
\end{aligned}
$$

where the inequalities are from Lemma 6.3 (22) and (27), respectively. Combining these observations, we obtain the result.

Lemma 6.6. Let $\mathbf{x}=\left(x_{1}, x_{2}, \ldots, x_{n}\right)$ and $\mathbf{x}^{\prime}=\left(x_{0}, x_{1}^{\prime}, \ldots, x_{n-1}^{\prime}\right)$ be $n$-chains with $x_{0} \in X_{v}$ and $x_{1} \in X_{\alpha(v)}$. If $\left(x_{0}, x_{1}\right)$ is a 2-chain and $d\left(x_{0}, x_{1}\right) \leq n$, then $\left\|\eta^{\mathbf{x}}-\eta^{\mathbf{x}^{\prime}}\right\| \leq \frac{\varepsilon}{R+1}$. 
Proof. Let $\bar{x}_{0}=f_{v}^{-1}\left(x_{1}\right)$ and set $\overline{\mathbf{x}}=\left(\bar{x}_{0}, x_{1}, \ldots, x_{n-1}\right)$. According to the definition (30) of $\eta^{\mathrm{x}}$ we have

$$
\begin{aligned}
& \eta^{\mathbf{x}}=\eta^{\left(x_{1}, x_{2}, \ldots, x_{n}\right)}=\frac{1}{\sqrt{n}}\left(\sum_{k=1}^{n-1} d_{k} \xi_{x_{k}}+d_{n} \xi_{x_{n}}\right), \\
& \eta^{\overline{\mathbf{x}}}=\eta^{\left(\bar{x}_{0}, x_{1}, \ldots, x_{n-1}\right)}=\frac{1}{\sqrt{n}}\left(\bar{d}_{0} \xi_{\bar{x}_{0}}+\sum_{k=1}^{n-1} \bar{d}_{k} \xi_{x_{k}}\right),
\end{aligned}
$$

where

$$
\begin{gathered}
d_{k}=c_{k-1}\left(x_{1}, \ldots, x_{k}\right)=\sqrt{\bar{a}_{1} \cdots \bar{a}_{k-1}\left(1+(n-k)\left(1-\bar{a}_{k}\right)\right)}, \\
\bar{d}_{k}=c_{k}\left(\bar{x}_{0}, x_{1}, \ldots, x_{k}\right)=\sqrt{\bar{a}_{0} \bar{a}_{1} \cdots \bar{a}_{k-1}\left(1+(n-k-1)\left(1-\bar{a}_{k}\right)\right)},
\end{gathered}
$$

with $\bar{a}_{0}=1, \bar{d}_{0}=1$ and $\bar{a}_{k}=a_{k}\left(\bar{x}_{0}, x_{1}, \ldots, x_{k}\right)=\min \left\{\left(1-\frac{\ln \left(1+\delta_{i}\right)}{N}\right)_{+}: 1 \leq i \leq k\right\}$.

For $1 \leq k \leq n-1$ we have either $1-\bar{a}_{k}=0$, hence $d_{k}-\bar{d}_{k}=0$, or

$$
\begin{aligned}
d_{k}-\bar{d}_{k} & =\frac{\sqrt{\bar{a}_{1} \cdots \bar{a}_{k-1}}\left(1-\bar{a}_{k}\right)}{\sqrt{1+(n-k)\left(1-\bar{a}_{k}\right)}+\sqrt{1+(n-k-1)\left(1-\bar{a}_{k}\right)}} \\
& \leq \frac{\left(1-\bar{a}_{k}\right)}{2 \sqrt{(n-k)\left(1-\bar{a}_{k}\right)}} \leq \frac{1}{2 \sqrt{n-k}} .
\end{aligned}
$$

Applying the above expressions for $\eta^{\mathbf{x}}$ and $\eta^{\overline{\mathbf{x}}}$ with this inequality, we estimate

$$
\begin{aligned}
\left\|\eta^{\overline{\mathbf{x}}}-\eta^{\mathbf{x}}\right\| & =\frac{1}{\sqrt{n}}\left\|\bar{d}_{0} \xi_{\bar{x}_{0}}+\sum_{k=1}^{n-1}\left(\bar{d}_{k}-d_{k}\right) \xi_{x_{k}}-d_{n} \xi_{x_{n}}\right\| \\
& \leq\left(\frac{2}{n}+\frac{1}{n} \sum_{k=1}^{n-1}\left(d_{k}-\bar{d}_{k}\right)^{2}\right)^{\frac{1}{2}} \\
& \leq\left(\frac{2}{n}+\frac{1}{4 n}\left(\frac{1}{n-1}+\frac{1}{n-2}+\cdots+1\right)\right)^{\frac{1}{2}} \\
& \leq\left(\frac{\ln n+9}{4 n}\right)^{\frac{1}{2}} \leq \frac{\varepsilon}{3(R+1)},
\end{aligned}
$$

where the final inequality comes from the choice (26) of $n$. Apply Lemma 6.5 to the chains $\mathbf{x}^{\prime}$ and $\overline{\mathbf{x}}$, noting that $d\left(x_{0}, \bar{x}_{0}\right)=d\left(x_{0}, x_{1}\right)-1 \leq n$, and use the previous inequality to conclude that

$$
\left\|\eta^{\mathbf{x}^{\prime}}-\eta^{\mathbf{x}}\right\| \leq\left\|\eta^{\mathbf{x}^{\prime}}-\eta^{\overline{\mathbf{x}}}\right\|+\left\|\eta^{\overline{\mathbf{x}}}-\eta^{\mathbf{x}}\right\| \leq \frac{2 \varepsilon}{3(R+1)}+\frac{\varepsilon}{3(R+1)}=\frac{\varepsilon}{R+1} .
$$

Lemma 6.7. Let $\mathbf{x}=\left(x_{0}, x_{1}, \ldots, x_{n-1}\right)$ and $\mathbf{x}^{\prime}=\left(x_{0}^{\prime}, x_{1}^{\prime}, \ldots, x_{n-1}^{\prime}\right)$ be $n$-chains in $X$. Then

$$
\left|\left\langle\eta^{\mathbf{x}}, \eta^{\mathbf{x}^{\prime}}\right\rangle\right| \leq \sup \left\{\left|\left\langle\xi_{y}, \xi_{y^{\prime}}\right\rangle\right|: y, y^{\prime} \in X_{v}, v \in V, d\left(y, y^{\prime}\right) \geq d\left(x_{0}, x_{0}^{\prime}\right)-6 n e^{N}\right\} .
$$

Proof. Let $k$ and $\ell$ be as in (13). Considering symmetry, we assume that $k \geq \ell$. Also, if $k \geq n$, then $\left\langle\eta^{\mathbf{x}}, \eta^{\mathbf{x}^{\prime}}\right\rangle=0$; so we assume that $k<n$. With these assumptions

$$
\left\langle\eta^{\mathbf{x}}, \eta^{\mathbf{x}^{\prime}}\right\rangle=\frac{1}{n} \sum_{i=0}^{n-k-1} c_{k+i} c_{\ell+i}^{\prime}\left\langle\xi_{x_{k+i}}, \xi_{x_{\ell+i}^{\prime}}\right\rangle .
$$


Making use of (21), we conclude that if $\theta_{k-1} \vee \theta_{\ell-1}^{\prime} \geq e^{N}-1$, then all the products $c_{k+i} c_{\ell+i}^{\prime}$ in (32) are zero, and we are done. Thus we assume that $\theta_{k-1} \vee \theta_{\ell-1}^{\prime} \leq$ $e^{N}-1$. Let $m$ be the largest number $0 \leq m \leq n-k-1$ with the property that $\theta_{k+m-1} \vee \theta_{\ell+m-1}^{\prime} \leq e^{N}-1$. By (17),

$$
d\left(x_{k+i}, x_{\ell+i}^{\prime}\right) \geq d\left(x_{k}, x_{\ell}^{\prime}\right)-2 n e^{N}
$$

for all $0 \leq i \leq m$. On the other hand, using (15),

$$
\begin{aligned}
d\left(x_{k}, x_{\ell}^{\prime}\right) & \geq d\left(x_{0}, x_{0}^{\prime}\right)-\sum_{i=0}^{k-1} d\left(x_{i}, x_{i+1}\right)-\sum_{j=0}^{\ell-1} d\left(x_{j}^{\prime}, x_{j+1}^{\prime}\right) \\
& \geq d\left(x_{0}, x_{0}^{\prime}\right)-\sum_{i=0}^{k-1}\left(\delta_{i}+2\right)-\sum_{j=0}^{\ell-1}\left(\delta_{j}^{\prime}+2\right) \\
& \geq d\left(x_{0}, x_{0}^{\prime}\right)-(k+\ell)\left(2\left(\theta_{k-1} \vee \theta_{\ell-1}^{\prime}\right)+2\right) \\
& \geq d\left(x_{0}, x_{0}^{\prime}\right)-4 n e^{N} .
\end{aligned}
$$

Combining these two inequalities, we obtain

$$
d\left(x_{k+i}, x_{\ell+i}^{\prime}\right) \geq d\left(x_{0}, x_{0}^{\prime}\right)-6 n e^{N}, \quad \text { for all } \quad 0 \leq i \leq m .
$$

Finally, arguing again on the basis of (21) as above, we conclude that the terms in (32) for $i>m$ are zero. Thus, applying (20) and the previous inequality, we see that

$$
\left|\left\langle\eta^{\mathbf{x}}, \eta^{\mathbf{x}^{\prime}}\right\rangle\right| \leq\left(\frac{1}{n} \sum_{i=0}^{m} c_{k+i} c_{\ell+i}^{\prime}\right) \cdot \sup _{\Omega}\left|\left\langle\xi_{y}, \xi_{y^{\prime}}\right\rangle\right| \leq \sup _{\Omega}\left|\left\langle\xi_{y}, \xi_{y^{\prime}}\right\rangle\right|,
$$

where $\Omega=\left\{\left(y, y^{\prime}\right): y, y^{\prime} \in X_{v}, v \in V, d\left(y, y^{\prime}\right) \geq d\left(x_{0}, x_{0}^{\prime}\right)-6 n e^{N}\right\}$.

Proposition 6.8. Given $R>0$ and $\varepsilon>0$, let $n, N$, and $\left(\xi_{x}\right)_{x \in X}$ be constructed as in the definition. For each $x_{0} \in X$, choose and fix an $n$-chain $\mathbf{x}=\left(x_{0}, x_{1}, \ldots, x_{n-1}\right)$ and consider the corresponding vector $\eta^{\mathbf{x}}=\eta^{\left(x_{0}, x_{1}, \ldots, x_{n-1}\right)}$. Then

$$
\left|\left\langle\eta^{\mathbf{x}}, \eta^{\mathbf{x}^{\prime}}\right\rangle\right| \leq \sup \left\{\left|\left\langle\xi_{y}, \xi_{y^{\prime}}\right\rangle\right|: d\left(y, y^{\prime}\right) \geq d\left(x_{0}, x_{0}^{\prime}\right)-6 n e^{N}, y, y^{\prime} \in X_{v}, v \in V\right\} .
$$

$$
\sup \left\{\left\|\eta^{\mathbf{x}}-\eta^{\mathbf{x}^{\prime}}\right\|: d\left(x_{0}, x_{0}^{\prime}\right)<R\right\} \leq \varepsilon,
$$

Proof. The support condition (34) was proven in Lemma 6.7. For the convergence condition (33) we show that for any $x_{0}, x_{0}^{\prime} \in X$ with $d\left(x_{0}, x_{0}^{\prime}\right)<R$ and any two $n$ chains $\mathbf{x}$ and $\mathbf{x}^{\prime}$ starting at $x_{0}$ and $x_{0}^{\prime}$, respectively, we have $\left\|\eta^{\mathbf{x}}-\eta^{\mathbf{x}^{\prime}}\right\| \leq \varepsilon$. Let $k$ and $\ell$ be as in (13) and (14). Let $\mathbf{x}(i)$ and $\mathbf{x}^{\prime}(j)$ be $n$-chains whose initial elements are the points $x_{i}, x_{j}^{\prime}$ given by Lemma 6.1, $0 \leq i \leq k, 0 \leq j \leq \ell$. Applying Lemmas 6.5 and 6.6 repeatedly (note that since $n>2^{R} R$ this is possible by Lemma 6.1), with the convention that all empty sums are zero, we have

$$
\begin{aligned}
\left\|\eta^{\mathbf{x}}-\eta^{\mathbf{x}^{\prime}}\right\| & \leq \sum_{i=0}^{k-1}\left\|\eta^{\mathbf{x}(i)}-\eta^{\mathbf{x}(i+1)}\right\|+\left\|\eta^{\mathbf{x}(k)}-\eta^{\mathbf{x}^{\prime}(\ell)}\right\|+\sum_{j=0}^{\ell-1}\left\|\eta^{\mathbf{x}^{\prime}(j)}-\eta^{\mathbf{x}^{\prime}(j+1)}\right\| \\
& \leq \frac{(k+\ell+1) \varepsilon}{R+1} \leq \varepsilon
\end{aligned}
$$


Conclusion of the proof of Theorem 5.3. Given $R>0$ and $\varepsilon>0$, let $x_{0}$ $\longmapsto \eta^{\left(x_{0}, x_{1}, \ldots, x_{n-1}\right)}$ be a map constructed as in Proposition 6.8. It satisfies the convergence and support conditions of Proposition [2.1] as shown by (33) and by (34) in conjunction with (29).

Let us note that Proposition 6.8 also reproves the following fact.

Theorem 6.9 (8], [19]). Let $A$ and $B$ be countable discrete groups and let $C$ be a common subgroup. If both $A$ and $B$ are exact, then the amalgamated free product $A *_{C} B$ is exact.

Proof. Given $R>0$ and $\varepsilon>0$, let $x_{0} \longmapsto \eta^{\left(x_{0}, x_{1}, \ldots, x_{n-1}\right)}$ be a map constructed as in Proposition 6.8. If the family $\left(\xi_{x}\right)_{x \in X}$ satisfies the support condition (ii) of Proposition 2.6 uniformly with respect to $v \in V$, then it follows from (34) that $\left\langle\eta^{\mathbf{x}}, \eta^{\mathbf{x}^{\prime}}\right\rangle=0$ whenever $d\left(x_{0}, x_{0}^{\prime}\right) \geq S+6 n e^{N}$. In particular, the map $x_{0} \longmapsto$ $\eta^{\left(x_{0}, x_{1}, \ldots, x_{n-1}\right)}$ satisfies the convergence and support conditions of Proposition 2.6 and $A *_{C} B$ is exact by Corollary 2.8 .

\section{Appendix}

We collect several elementary results on the construction of metrics required for our treatment of amalgamated free products. Let $X$ be a set. A partial metric on $X$ is an integer-valued function $\widehat{d}: \mathcal{D} \rightarrow \mathbb{R}_{+}$defined on a domain $\mathcal{D} \subset X \times X$ and satisfying, for all $x, y \in X$,

(i) $\widehat{d}(x, y)=\widehat{d}(y, x)$, and

(ii) $\widehat{d}(x, y)=0$ if and only if $x=y$.

In these statements it is assumed that all relevant pairs belong to the domain $\mathcal{D}$ of $\widehat{d}$ and that the domain $\mathcal{D}$ is symmetric and contains the diagonal.

We now associate a metric to a partial metric. The construction is analogous to that of path metrics; intuitively the distance between two points is the length of the shortest path between them. A path from $x$ to $y \in X$ is a sequence $x=$ $x_{0}, x_{1}, \ldots, x_{n}=y$ such that for every $1 \leq j \leq n$ we have $\left(x_{i-1}, x_{i}\right) \in \mathcal{D}$. The length of a path is $\sum_{i=1}^{n} \widehat{d}\left(x_{i-1}, x_{i}\right)$. The domain $\mathcal{D}$ is ample if for every $x, y \in \mathcal{D}$ there exists a path from $x$ to $y$.

Proposition 7.1. Let $\widehat{d}$ be a partial metric on $X$, defined on an ample domain $\mathcal{D}$. Define, for all $x, y \in X$,

$$
d(x, y)=\inf \{\text { length of paths from } x \text { to } y\} .
$$

Then $d$ is an integer-valued metric on $X$.

The metric $d$ defined in the proposition is the metric envelope of $\widehat{d}$.

Example. If $X$ is the vertex set of a connected graph and $\widehat{d}$ is the constant 1 on the domain of all pairs $(x, y)$ that represent edges, then $d$ is the path metric; $d(x, y)$ is the smallest number of edges on a path from $x$ to $y$.

Remark. If $(x, y) \in \mathcal{D}$, then $d(x, y)=\widehat{d}(x, y)$ if and only if the length of every path from $x$ to $y$ is greater than or equal to $\widehat{d}(x, y)$. If $\mathcal{D}=X \times X$ and $\widehat{d}$ is a metric, then $d=\widehat{d}$.

Proposition 7.2. Let $\widehat{d}$ be a partial metric on $X$ and let $\varphi: X \rightarrow X$ be a bijection with the property that

(i) $(x, y) \in \mathcal{D}$ if and only if $(\varphi(x), \varphi(y)) \in \mathcal{D}$, and

(ii) $\widehat{d}(\varphi(x), \varphi(y))=\widehat{d}(x, y)$ for all $(x, y) \in \mathcal{D}$. 
Then $\varphi$ is an isometry for the metric envelope.

Proof. It suffices to show that

$$
d(\varphi(x), \varphi(y)) \leq d(x, y), \quad \text { for all } x, y \in X .
$$

Let $\varepsilon>0$ be given. Let $\left(x_{0} \ldots, x_{n}\right)$ be a path in $X$ from $x$ to $y$ such that $\sum_{j=1}^{n} \widehat{d}\left(x_{j-1}, x_{j}\right)<d(x, y)+\varepsilon$. Then $\left(\varphi\left(x_{0}\right), \ldots, \varphi\left(x_{n}\right)\right)$ is a path from $\varphi(x)$ to $\varphi(y)$, and we have

$$
d(\varphi(x), \varphi(y)) \leq \sum_{i=1}^{n} \widehat{d}\left(\varphi\left(x_{i-1}\right), \varphi\left(x_{i}\right)\right)=\sum_{j=1}^{n} \widehat{d}\left(x_{j-1}, x_{j}\right)<d(x, y)+\varepsilon .
$$

Since $\varepsilon>0$ was arbitrary, we are done.

\section{REFERENCES}

[1] J. Alonso and M. Bridson, Semihyperbolic groups, Proc. London Math. Soc. 70 (1995), 56114. MR 95j:20033

[2] C. Anantharaman-Delaroche, Amenability and exactness for dynamical systems and their $C^{*}$-algebras, Trans. Amer. Math. Soc. 354 (2002), 4153-4178.

[3] C. Anantharaman-Delaroche and J. Renault, Amenable groupoids, with a foreword by Georges Skandalis and Appendix B by E. Germain, Monographies de L'Enseignement Math., vol. 36, L'Enseignement Math., Geneva, 2000. MR 2001m:22005

[4] G. Baumslag, Topics in combinatorial group theory, ETH Lectures in Mathematics, Birkhäuser-Verlag, Basel, 1993. MR 94j:20034

[5] X. Chen, M. Dadarlat, E. Guentner, and G. Yu, Uniform embeddings into Hilbert space and free products of groups, to appear in J. Funct. Anal.

[6] P. Cherix, M. Cowling, P. Jolissaint, P. Julg, and A. Valette, Groups with the Haagerup property. Gromov's a-T-menability, Progress in Mathematics, vol. 197, Birkhäuser-Verlag, Basel, 2001. MR 2002h:22007

[7] A. Dranishnikov, G. Gong, V. Lafforgue, and G. Yu, Uniform embeddings into Hilbert space and a question of Gromov, Canad. Math. Bull. 45 (2002), 60-70. MR 2003a:57043

[8] K. Dykema, Exactness of reduced amalgamated free product $C^{*}$-algebras, Preprint, 1999.

[9] $ـ$ Free products of exact groups, $C^{*}$-algebras (Münster, 1999) (J. Cuntz and S. Echterhoff, eds.), Springer-Verlag, Berlin, 2000, pp. 61-70. MR 2001m:46133

[10] S. Ferry, A. Ranicki, and J. Rosenberg (eds.), Novikov conjectures, index theorems and rigidity, London Mathematical Society Lecture Notes, nos. 226, 227, Cambridge University Press, 1995. MR 96m:57002 MR 96m:57003

[11] S. Gersten, Bounded cocycles and combings of groups, unpublished manuscript, version 5.5, 1991, cf. Internat. J. Algebra Comput. 2 (1992), 307-326. MR 93i:20029

[12] M. Gromov, Asymptotic invariants of infinite groups, London Mathematical Society Lecture Notes, no. 182, pp. 1-295, Cambridge University Press, Cambridge, 1993. MR 95m:20041

[13] E. Guentner and J. Kaminker, Exactness and the Novikov conjecture, Topology 41 (2002), no. 2, 411-418. MR 2003e:46097a

[14] E. Kirchberg and S. Wassermann, Exact groups and continuous bundles of $C^{*}$-algebras, Mathematische Annalen 315 (1999), 169-203. MR 2000i:46050

[15] _ Permanence properties of $C^{*}$-exact groups, Documenta Mathematica 4 (1999), 513558 (electronic). MR 2001i:46089

[16] N. Ozawa, Amenable actions and exactness for discrete groups, C. R. Acad. Sci. Paris Sér. I Math. 330 (2000), no. 8, 691-695. MR 2001g:22007

[17] J. P. Serre, Trees, Springer-Verlag, New York, 1980, Translation from French of "Arbres, Amalgames, $S L_{2}$ ", Astérisque no. 46. MR 82c:20083

[18] G. Skandalis, J. L. Tu, and G. Yu, The coarse Baum-Connes conjecture and groupoids, Topology 41 (2002), 807-834. MR 2003c:58020

[19] J. L. Tu, Remarks on Yu's Property A for discrete metric spaces and groups, Bull. Soc. Math. France 129 (2001), 115-139. MR 2002j:58038 
[20] G. Yu, The Coarse Baum-Connes conjecture for spaces which admit a uniform embedding into Hilbert space, Inventiones Math. 139 (2000), 201-240. MR 2000j:19005

Department of Mathematics, Purdue University, 1395 Mathematical Sciences BuildING, West Lafayette, Indiana 47907-1395

E-mail address: mdd@math.purdue.edu

Mathematics Department, University of Hawail, Manoa, 2565 McCarthy Mall, HonOlulu, HaWail 96822

E-mail address: erik@math.hawaii.edu 